\title{
Character Recognition And Extraction of An Indian State License Plates Using K-NN
}

A Project Report Submitted In Partial Fulfillment of the Requirements for the Award of Degree

\section{BACHELOR OF TECHNOLOGY}

In

\section{ELECTRONICS AND COMMUNICATION ENGINEERING}

\author{
Submitted By \\ Rajasekhar Ponakala (13011M3442) \\ Hari Krishna Adda (130M3420) \\ Ch. Aravind Kumar (13011M3402) \\ Kavya Avula (13011M3443) \\ Under the esteemed guidance of \\ Prof. K. Anitha Sheela

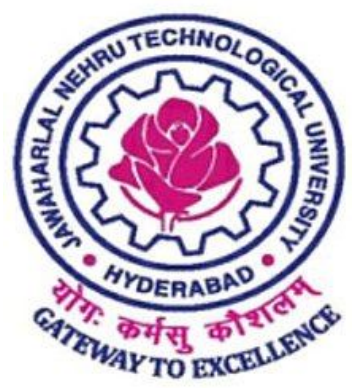

Department of Electronics and Communication Engineering JAWAHARLAL NEHRU TECHNOLOGICAL UNIVERSITY HYDERABAD JNTUH - College of Engineering Hyderabad (Autonomous)

(Hyderabad 500085 - Telangana - India)

December - 2016 


\section{ABSTRACT}

License plate recognition is an application-specific optimisation in Optical Character Recognition (OCR) software which enables computer systems to read automatically the License Plates of vehicles from digital images.

This thesis discusses about the character extraction from the respective License Plates of vehicles and problems in the character extraction process. An OCR based training algorithm named k-nearest neighbor with predefined OpenCV libraries are implemented and evaluated in the BeagleBone Black Open Hardware.

In an OCR, the character extraction involves certain steps which includes Image acquisition, Pre-processing, Feature extraction, Detection/ Segmentation, High-level processing, Decision making. A key advantage of the method is that it is a fairly straightforward technique which utilises from k-nearest neighbor algorithm segments normalized result as a format in text.

The results show that, training an image with this algorithm gives better results when compared with other algorithms. 


\section{CHAPTER 1 - INTRODUCTION}

\subsection{Introduction}

Optical Character Recognition, or OCR, is a technology that enables people to convert different types of documents, such as scanned paper documents, PDF files or images captured by a digital camera into editable and searchable data.

Imagine there is a paper document - for example, magazine article, brochure, or PDF contract a person sent to another person by email. Obviously, a scanner is not enough to make this information available for editing, say in Microsoft Word. All a scanner can do is create an image or a snapshot of the document that is nothing more than a collection of black and white or colour dots, known as a raster image. In order to extract and repurpose data from scanned documents, camera images or image-only PDFs, it needs an OCR software that would single out letters on the image, put them into words and then - words into sentences, thus enabling people to access and edit the content of the original document.

\subsection{Research fields and Applications of OCR}

OCR is a field of research in pattern recognition, artificial intelligence and computer vision. OCR engines have been developed into many kinds of domain-specific OCR applications, such as receipt OCR, invoice OCR, check OCR, legal billing document OCR. They can also be used for Automatic number plate recognition.

\subsubsection{Application-specific optimisations:}

In recent years, the major OCR technology providers began to tweak OCR systems to better deal with specific types of input. Beyond an application-specific lexicon, better performance can be had by taking into account business rules, standard expression, or rich information contained in color images. This strategy is called "Application-Oriented OCR" or "Customised OCR", and has been applied to OCR of license plates, business cards, invoices, 
screenshots, ID cards, driver licenses, and automobile manufacturing. This project is mainly focussed on OCR of license plates.

\subsection{Computer Vision Library}

OpenCV (Open Source Computer Vision) is a library of programming functions mainly aimed at real-time computer vision and also a machine learning software library. Opencv was built to provide a common infrastructure for computer vision applications and to accelerate the use of machine perception in the commercial products. Being a BSD-licensed product, OpenCV makes it easy for businesses to utilize and modify the code. In this project OpenCV includes a statistical machine learning library that contains k-nearest neighbor algorithm which is coded in Python (a widely used dynamic programming language).

\subsection{Open Hardware}

There are many definitions for open source hardware. The general norm is that Open Source hardware is electronic hardware design that is freely available under one of the recognized open source licenses. The open source hardware includes schematics, diagrams and design rules that can be used, studied and modified without restriction and can be copied and redistributed in modified or unmodified form either without restriction or with minimal restrictions only to ensure that further recipients can do the same.

Open-source hardware was preceded, influenced and shaped by several prominent cases in which important technologies were developed collaboratively and out in the open. Its historical antecedents include the open source and free software movements, from which it derived its principles. Despite the deep roots of these legacies, open source hardware only became known as such in the last decade. This was mostly due to the rise of the internet, which made sharing hardware designs possible, the commercial success of open source software, which gave it public visibility, and the decrease in cost of production tools, which made it feasible.

There are many types of Open Hardwares available. This project is implemented and tested on BeagleBone Black (a type of Open Hardware). BeagleBone Black is a low-cost, 
community-supported development platform for developers and hobbyists. It boots Linux in under 10 seconds and get started on development in less than 5 minutes with just a single USB cable.

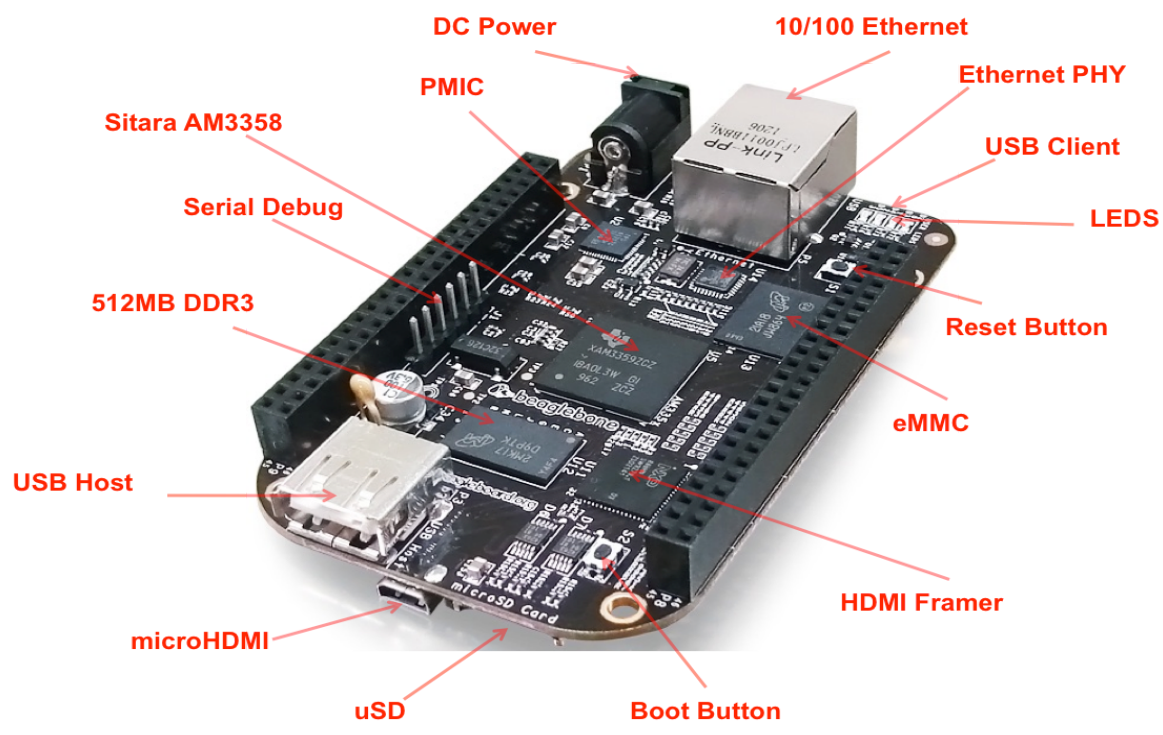

Fig 1 BeagleBone Black

\subsection{Aim, Objectives and Approach of the Project}

\subsubsection{Aim:}

Main Aim of this project is to develop a License Plate Recognition software which recognises different types of characters in an image and gives specific extracted output in text format.

\subsubsection{Objectives:}

The objective of this project is to extract characters from a License Plate with the use of computer vision libraries and algorithms. A suitable algorithm needs to be constructed and trained for different set of images. The program code has to be written in Python with the inclusion of suitable OpenCV libraries. The program should be able to extract the characters and print the output in text format for training purpose. 


\subsubsection{Approach:}

To solve the defined character recognition process the python coded algorithm with OpenCV libraries is used. The processing of code is divided into the next categories:

- Image acquisition

- Pre-processing

- Feature extraction

- Detection/segmentation

- High-level processing

- Decision making

\subsection{Organization of thesis}

Chapter 2 explains about concepts of License Plate Recognition and algorithm.

Chapter 3 will explain about the step by step process done in extraction of License Plate.

Chapter 4 will have the Conclusion and Future scope of the Project implemented. 


\section{CHAPTER 2 - DESCRIPTION OF OCR AND ALGORITHM}

\subsection{OCR on License Plates}

License plate recognition is a technology that uses optical character recognition on images to read vehicle registration plates. It can use existing closed-circuit television, road-rule enforcement cameras, or cameras specifically designed for the task. It is used by police forces around the world for law enforcement purposes, including to check if a vehicle is registered or licensed. It is also used for electronic toll collection on pay-per-use roads and as a method of cataloging the movements of traffic for example by highways agencies.

License Plate recognition can be used to store the images captured by the cameras as well as the text from the license plate, with some configurable to store a photograph of the driver. Systems commonly use infrared lighting to allow the camera to take the picture at any time of the day. This technology must take into account plate variations from place to place.

Concerns about these systems have centered on privacy fears of government tracking citizens' movements, misidentification, high error rates, and increased government spending. Critics have described it as a form of mass surveillance.

\subsection{OpenCV}

OpenCV (Open Source Computer Vision Library) is an open source computer vision and machine learning software library. OpenCV was built to provide a common infrastructure for computer vision applications. Being a BSD-licensed product, OpenCV makes it easy for anyone to utilize and modify the code.

The library has more than 2500 optimized algorithms, which includes a comprehensive set of both classic and state-of-the-art computer vision and machine learning algorithms. These algorithms can be used to detect and recognize faces, identify objects, identify Characters. It has $\mathrm{C}++, \mathrm{C}$, Python, Java and MATLAB interfaces and supports Windows, Linux, Android and Mac OS. 
OpenCV-Python is a library of Python bindings designed to solve computer vision problems. OpenCV-Python makes use of Numpy, which is a highly optimized library for numerical operations with a MATLAB-style syntax. All the OpenCV array structures are converted to and from Numpy arrays.

\section{$2.3 k$-nearest neighbors algorithm $(k-N N)$}

OpenCV includes a statistical machine learning library that contains k-nearest neighbor algorithm which is coded in Python (a widely used dynamic programming language).

In pattern recognition, the $k$-Nearest Neighbors algorithm (or $k$-NN for short) is a non-parametric method used for classification and regression. In both cases, the input consists of the $k$ closest training examples in the feature space. The output depends on whether $k$-NN is used for classification or regression:

In $k-N N$ classification, the output is a class membership. An object is classified by a majority vote of its neighbors, with the object being assigned to the class most common among its $k$ nearest neighbors ( $k$ is a positive integer, typically small). If $k=1$, then the object is simply assigned to the class of that single nearest neighbor. In $k-N N$ regression, the output is the property value for the object. This value is the average of the values of its $k$ nearest neighbors.

$k$-NN is a type of instance-based learning, or lazy learning, where the function is only approximated locally and all computation is deferred until classification. The $k$-NN algorithm is among the simplest of all machine learning algorithms.

Both for classification and regression, it can be useful to assign weight to the contributions of the neighbors, so that the nearer neighbors contribute more to the average than the more distant ones. For example, a common weighting scheme consists in giving each neighbor a weight of $1 / d$, where $d$ is the distance to the neighbor. The neighbors are taken from a set of objects for which the class (for $k$-NN classification) or the object property value (for $k$-NN regression) is known. This can be thought of as the training set for the algorithm, though no explicit training step is required. 
A shortcoming of the $k$-NN algorithm is that it is sensitive to the local structure of the data. The algorithm is not to be confused with $k$-means, another popular machine learning technique.

\subsubsection{Concept of Algorithm}

The training examples are vectors in a multidimensional feature space, each with a class label. The training phase of the algorithm consists only of storing the feature vectors and class labels of the training samples.

In the classification phase, $k$ is a user-defined constant, and an unlabeled vector (a query or test point) is classified by assigning the label which is most frequent among the $k$ training samples nearest to that query point.

A commonly used distance metric for continuous variables is Euclidean distance. For discrete variables, such as for text classification, another metric can be used, such as the overlap metric (or Hamming distance). In the context of gene expression microarray data, for example, $k$-NN has also been employed with correlation coefficients such as Pearson and Spearman. Often, the classification accuracy of $k$-NN can be improved significantly if the distance metric is learned with specialized algorithms such as Large Margin Nearest Neighbor or Neighbourhood components analysis.

A drawback of the basic "majority voting" classification occurs when the class distribution is skewed. That is, examples of a more frequent class tend to dominate the prediction of the new example, because they tend to be common among the $k$ nearest neighbors due to their large number. One way to overcome this problem is to weight the classification, taking into account the distance from the test point to each of its $k$ nearest neighbors. The class (or value, in regression problems) of each of the $k$ nearest points is multiplied by a weight proportional to the inverse of the distance from that point to the test point. Another way to overcome skew is by abstraction in data representation. For example, in a self-organizing map (SOM), each node is a representative (a center) of a cluster of similar points, regardless of their density in the original training data. $K$-NN can then be applied to the SOM. 


\subsubsection{K-NN Feature extraction}

When the input data to an algorithm is too large to be processed and it is suspected to be redundant (e.g. the same measurement in both feet and meters) then the input data will be transformed into a reduced representation set of features (also named features vector). Transforming the input data into the set of features is called feature extraction. If the features extracted are carefully chosen it is expected that the features set will extract the relevant information from the input data in order to perform the desired task using this reduced representation instead of the full size input. Feature extraction is performed on raw data prior to applying $k$-NN algorithm on the transformed data in feature space.

An example of a typical computer vision computation pipeline for face recognition using $k$-NN including feature extraction and dimension reduction pre-processing steps (usually implemented with OpenCV):

- Haar face detection

- Mean-shift tracking analysis

- PCA or Fisher LDA projection into feature space, followed by $k$-NN classification 


\section{CHAPTER 3 - Processing Steps in Character Recognition}

The organization of a computer vision system is highly application dependent. Some systems are stand-alone applications which solve a specific measurement or detection problem. The specific implementation of a computer vision system also depends on if its functionality is pre-specified or if some part of it can be learned or modified during operation. Many functions are unique to the application. There are, however, typical functions which are found in many computer vision systems.

The following are the steps involved in extraction of characters from LIcense plate.

\subsection{Image acquisition}

A digital image is produced by one or several image sensors i.e light-sensitive cameras.

The pixel values typically correspond to light intensity in one or several spectral bands (gray images or colour images), but can also be related to various physical measures, such as depth, absorption or reflectance of sonic or electromagnetic waves.

Fig-2 is captured using webcam.

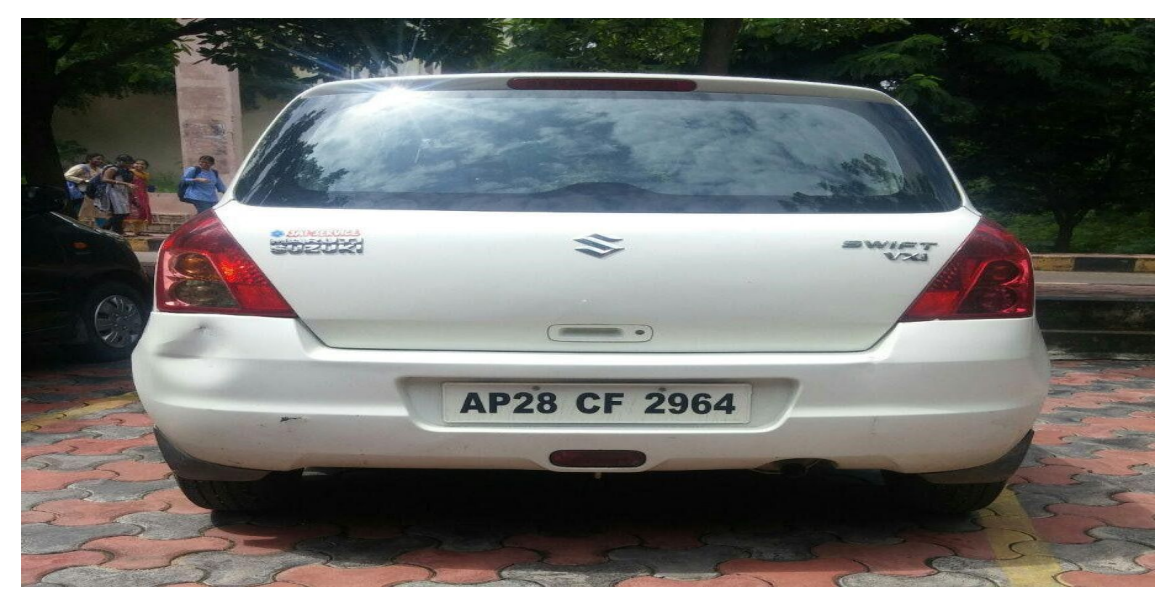

\section{AP28 CF 2964}

Fig-2 Original Scene 


\subsection{Pre-processing}

Before a computer vision method can be applied to image data in order to extract some specific piece of information, it is usually necessary to process the data in order to assure that it satisfies certain assumptions implied by the following methods,

- Noise reduction in order to assure that sensor noise does not introduce false information.

- Contrast enhancement to assure that relevant information can be detected.

- Re-sampling in order to assure that the image coordinate system is correct.

- Scale space representation to enhance image structures at locally appropriate scales.

From the previous image, the image is converted to Grayscale(Fig-3) and further to threshold(Fig-4,5) to reduce noise.
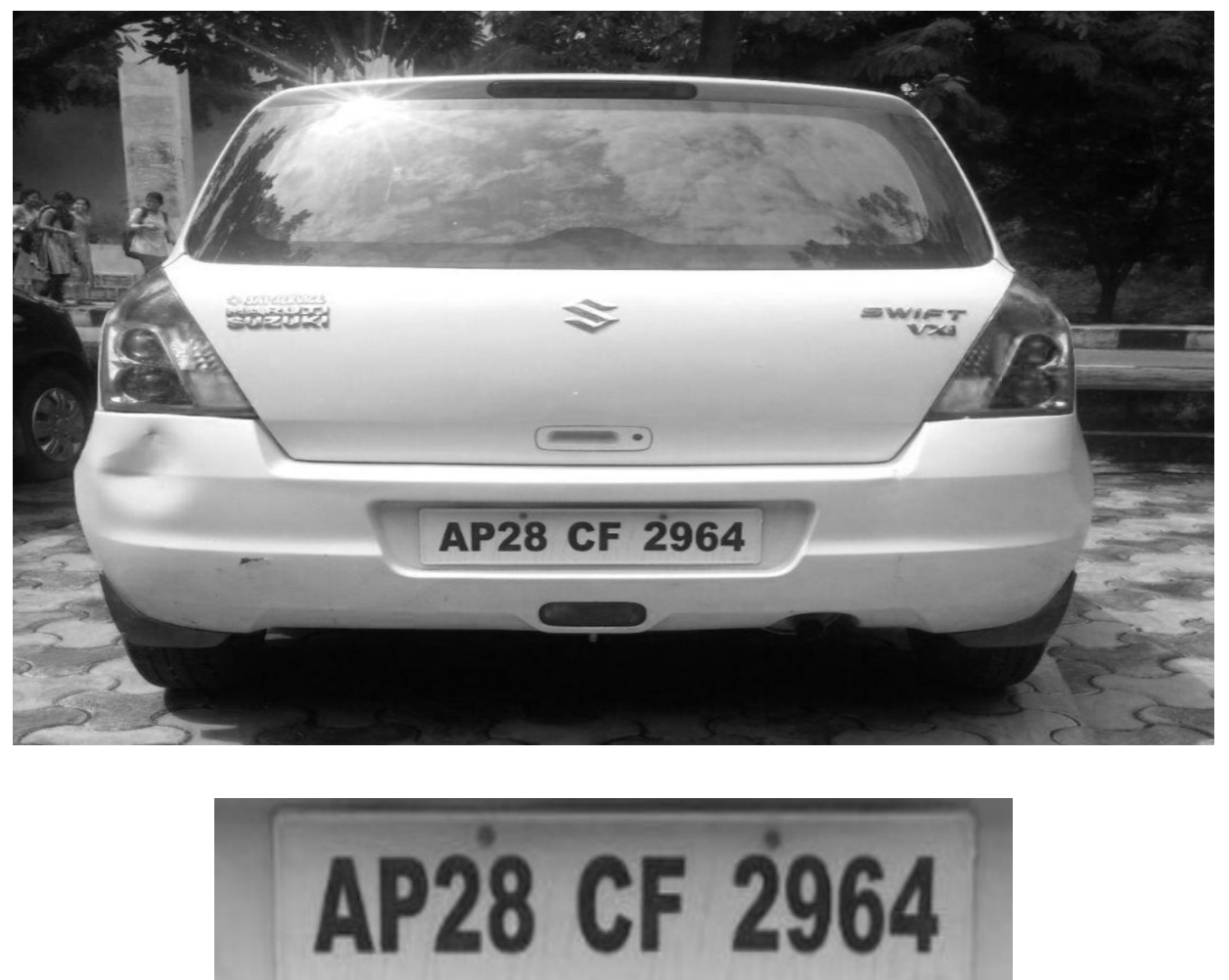

Fig-3 Grayscale Image 


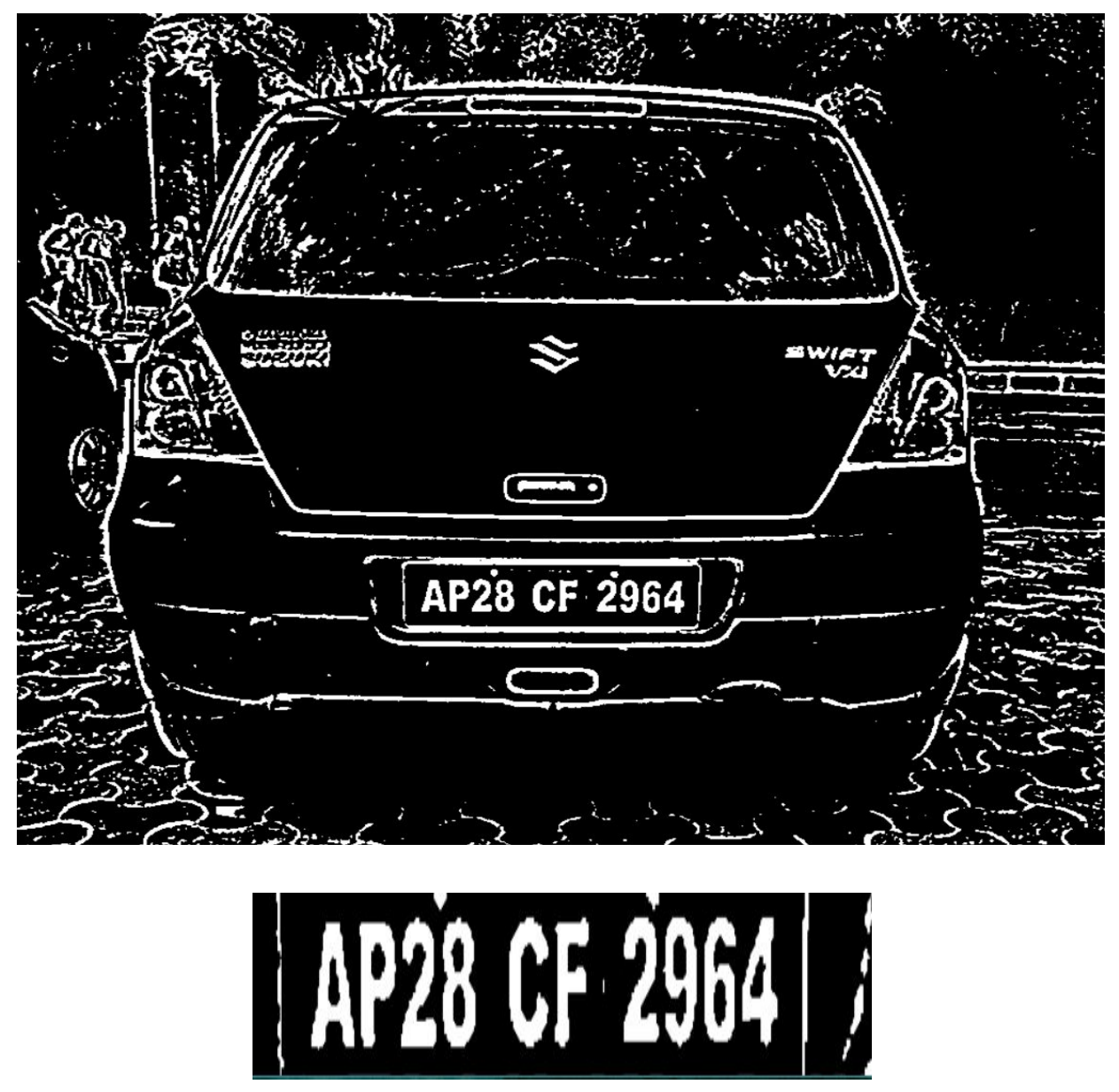

Fig-4 Threshold Image1 


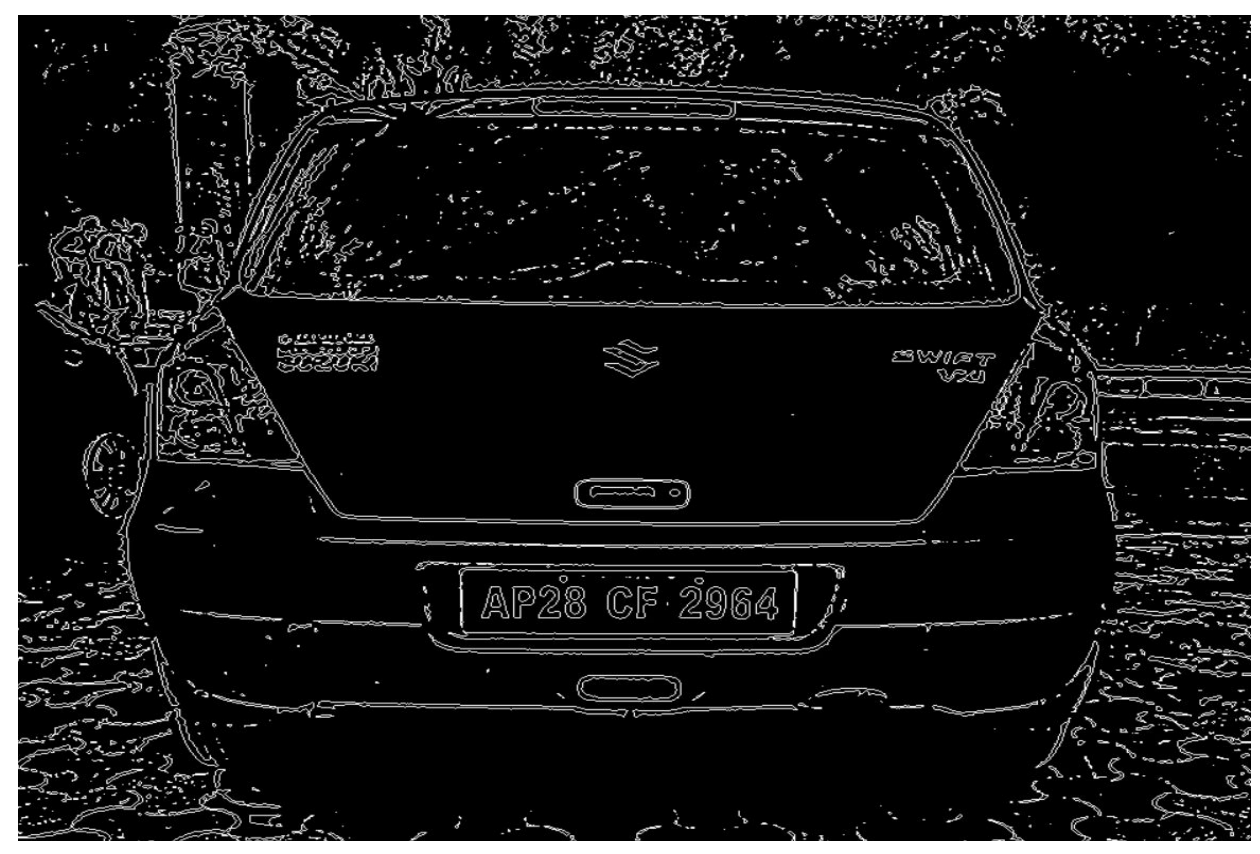

Fig-5 Threshold Image2

\subsection{Feature extraction}

Image features at various levels of complexity are extracted from the image data. Typical examples of such features are:

- Lines, edges and ridges.

- Localized interest points such as corners, points, etc.

- More complex features may be related to texture, shape or motion.

From threshold image, features like lines, edges, ridges etc are separated to identify possible characters(Fig-6). 


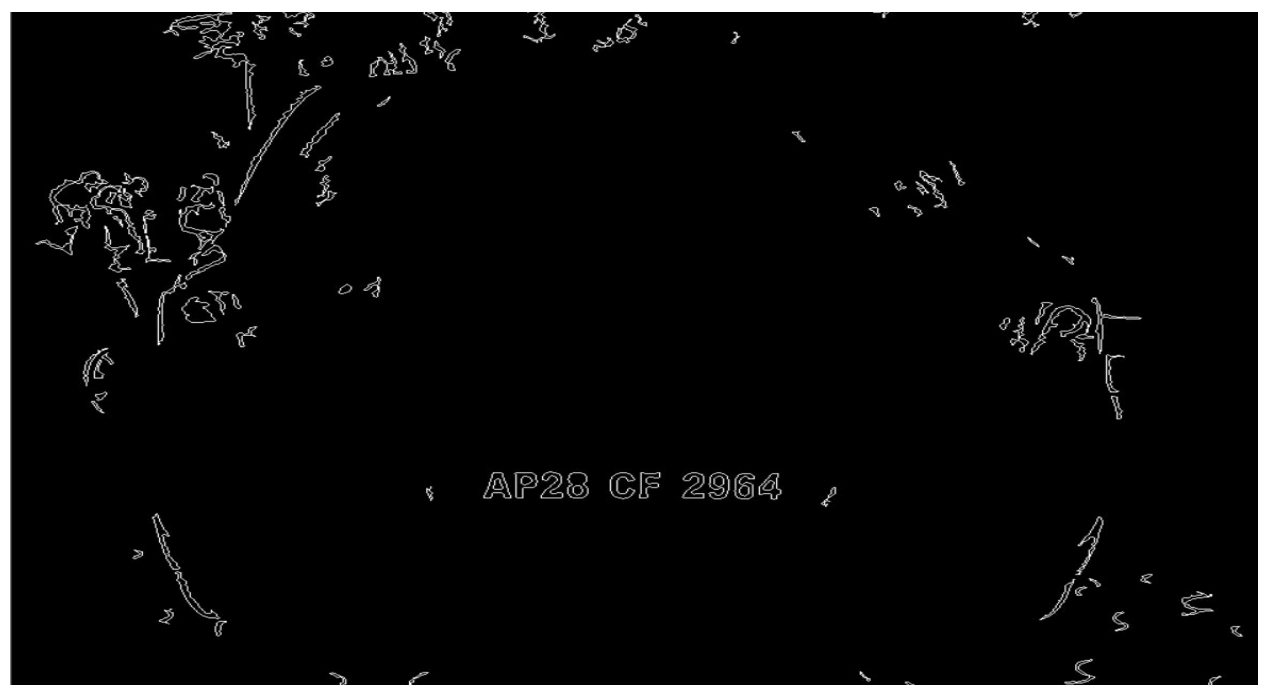

Fig-6 Possible Characters

\subsection{Detection/segmentation}

At some point in the processing a decision is made about which image points or regions of the image are relevant for further processing. For example

- Selection of a specific set of interest points

- Segmentation of one or multiple image regions which contain a specific object of interest.

In Fig-7, possible characters are matched with trained characters.

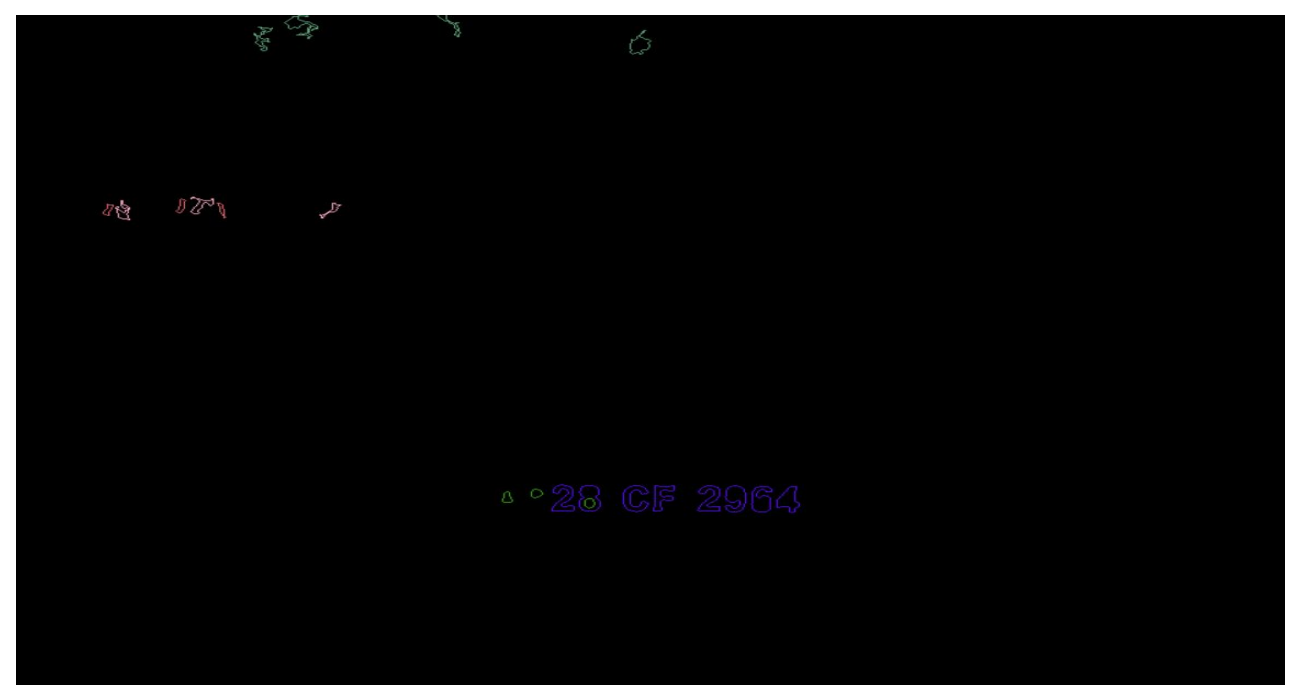

Fig-7 Matching Characters in the Scene 


\subsection{High-level processing}

At this step the input is typically a small set of data, a set of points or an image region which is assumed to contain a specific object. The remaining processing deals with

- Verification of data that satisfy model-based and application specific assumptions.

- Estimation of application specific parameters, such as object pose or object size.

- Image recognition - classifying a detected object into different categories.

- Image registration - comparing and combining two different views of the same object.

In Fig- 8 all the possible plates are identified and treated as rectangles, the rectangles which does not contain accurate characters are discarded.

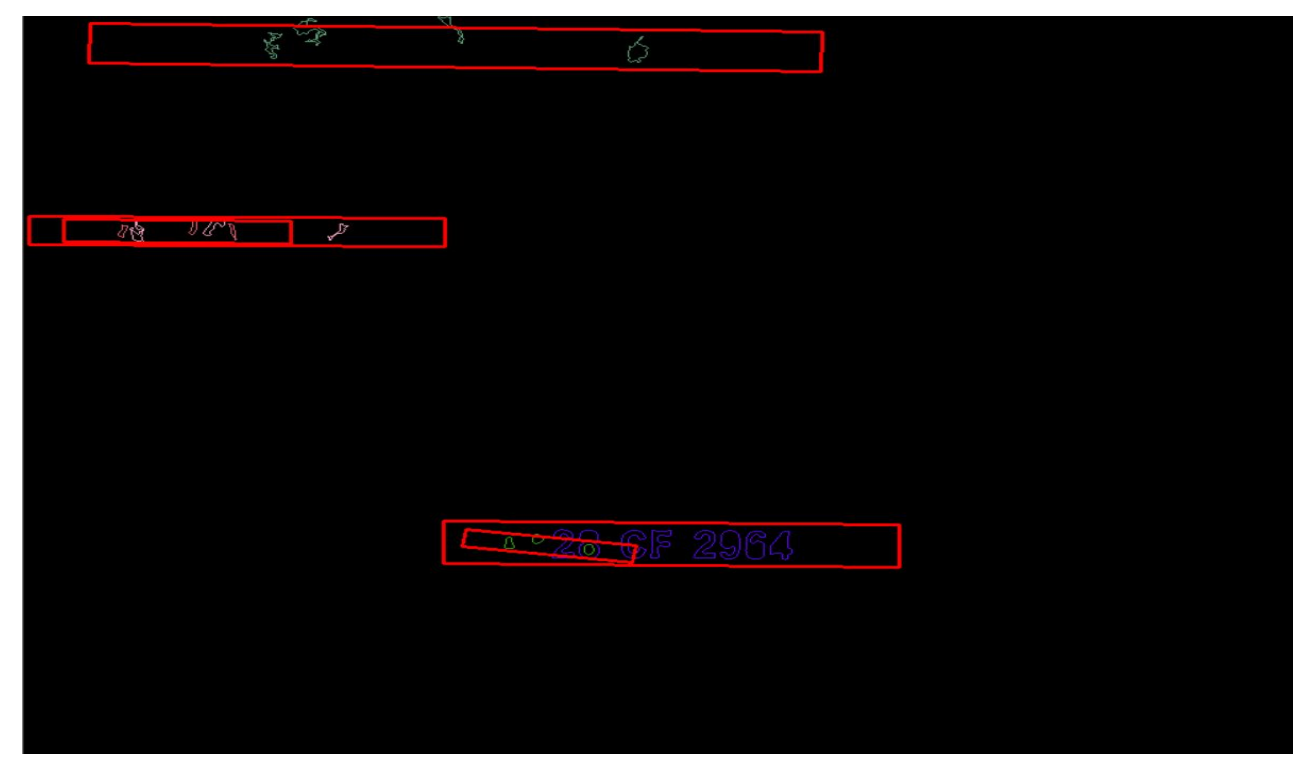

Fig-8 Possible Plates in the Scene 


\subsection{Decision making}

Making the final decision required for the following applications:

- Pass/fail on automatic inspection.

- Match / no-match in recognition.

The final rectangle(Fig-9) with appropriate characters from previous stage is matched with the trained characters and printed on the original image(as shown in Fig-10).

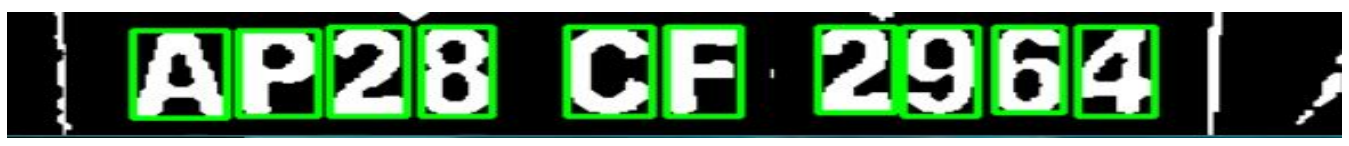

Fig-9 Plate with most recognised characters

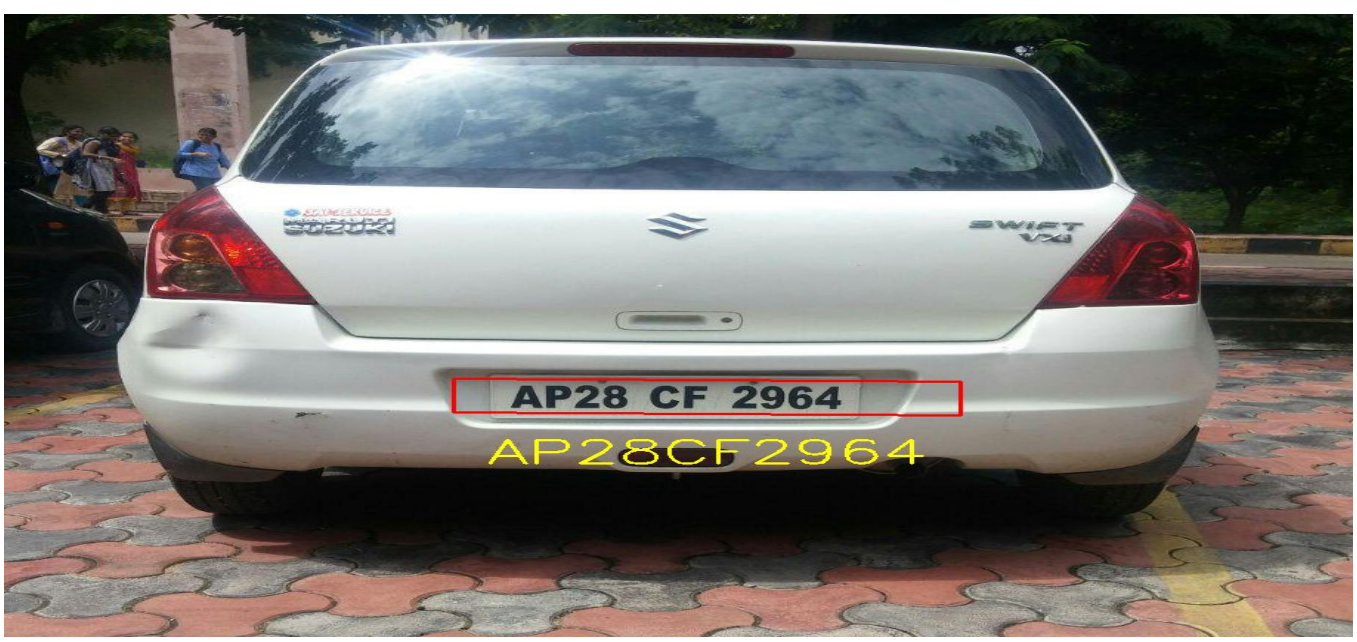

Fig-10 Recognised Characters is printed on original Image 


\section{CHAPTER 4 - CONCLUSION \& FUTURE SCOPE}

\section{CONCLUSION:}

This Project provides an implementation of License Plate Recognition using Optical Character Recognition(OCR) which is an electronic conversion of images of typed, handwritten or printed text into machine-encoded text. The project was able to study and resolve algorithmic and mathematical aspects of the License Plate Recognition systems, such as problematic of computer vision, pattern recognition, OCR \& Neural networks.

License Plate Recognition solution has been tested on static snapshots of vehicles, which has been divided into several sets according to difficulty. Sets of blurry and skewed snapshots give worse recognition rates than a set of snapshots which has been captured clearly. The main objective of this project was not to find a one hundred percent recognizable set of snapshots, but to test the invariance of the algorithms on random snapshots systematically classified to the sets according to their properties.

Source code obtained from different references could extract the characters up to only 5 in number, Improvisation of this source code is done to extract more than 8 characters to reach indian standards. This is achieved by changing the dimensions of best possible outcome of license plate region. 
Some random tests are performed on different images containing License Plate, results are shown below

\section{Test 1:}

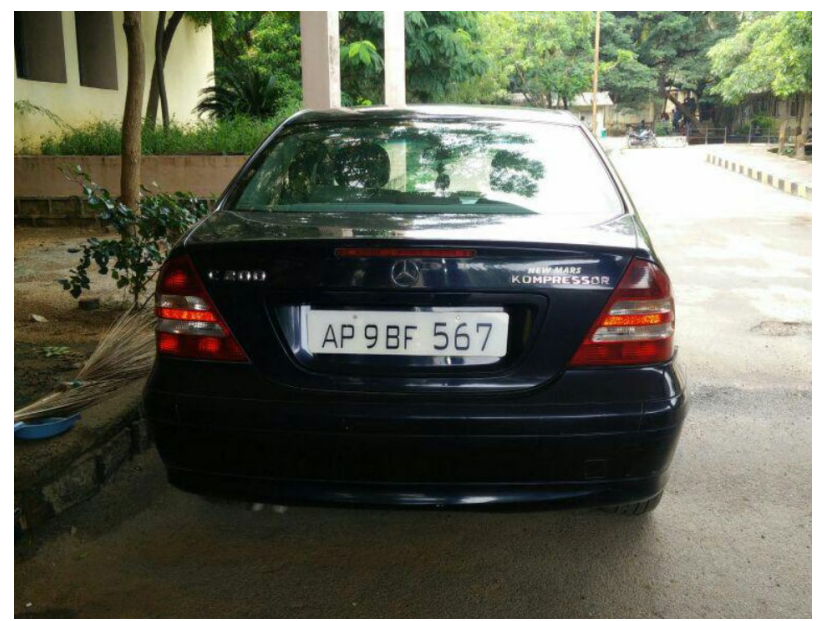

Fig-11

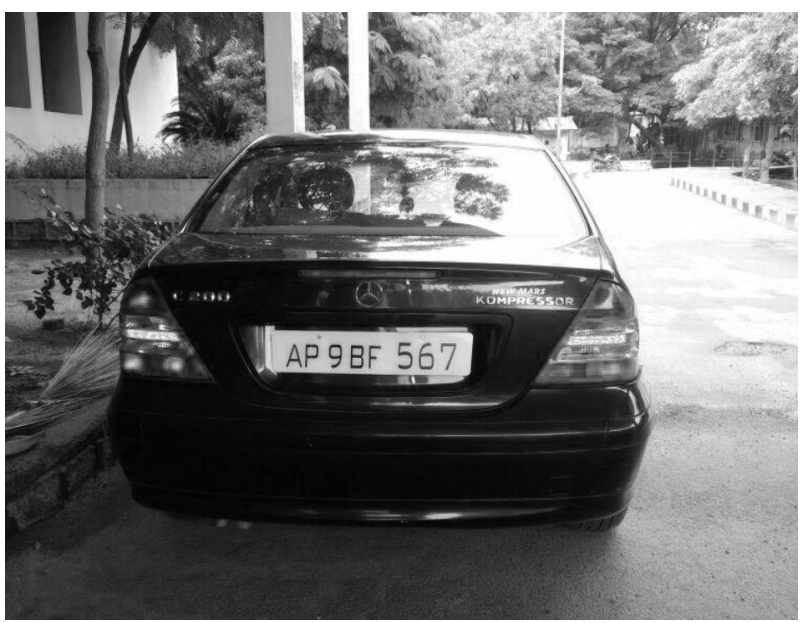

Fig-12

Fig-11 is original scene captured from webcam, From this image it is converted to Grayscale (Fig-12)

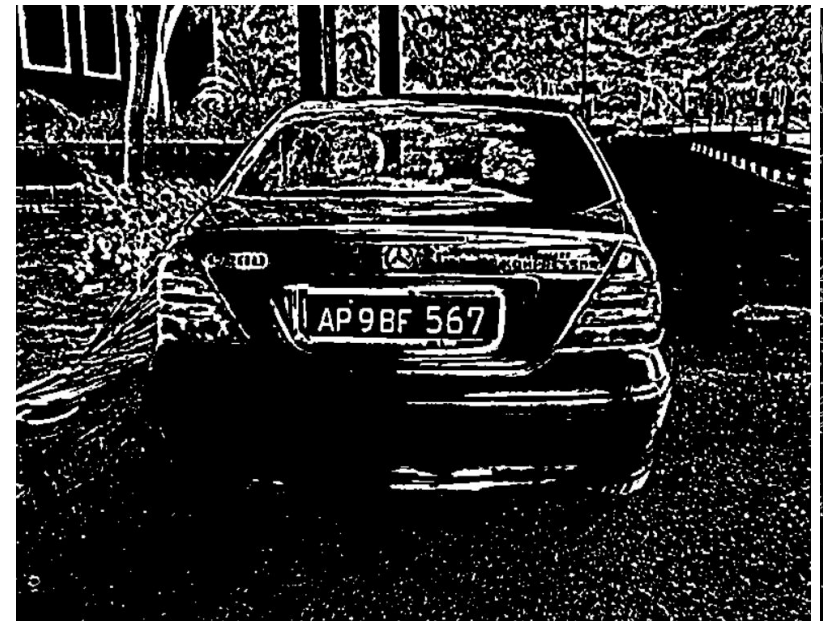

Fig-13

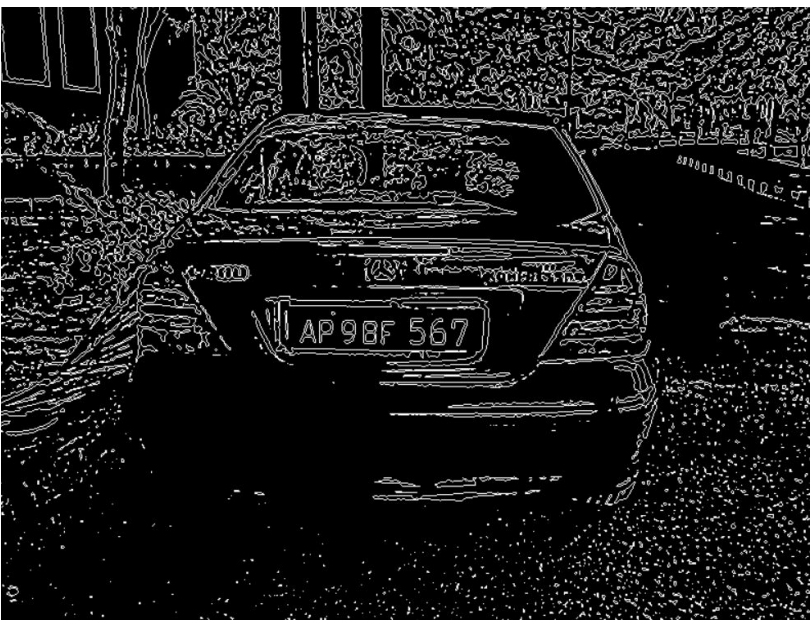

Fig-14 
Threshold image(Fig-13) is obtained from grayscale image and it is further processed to to reduce noise(Fig-14).

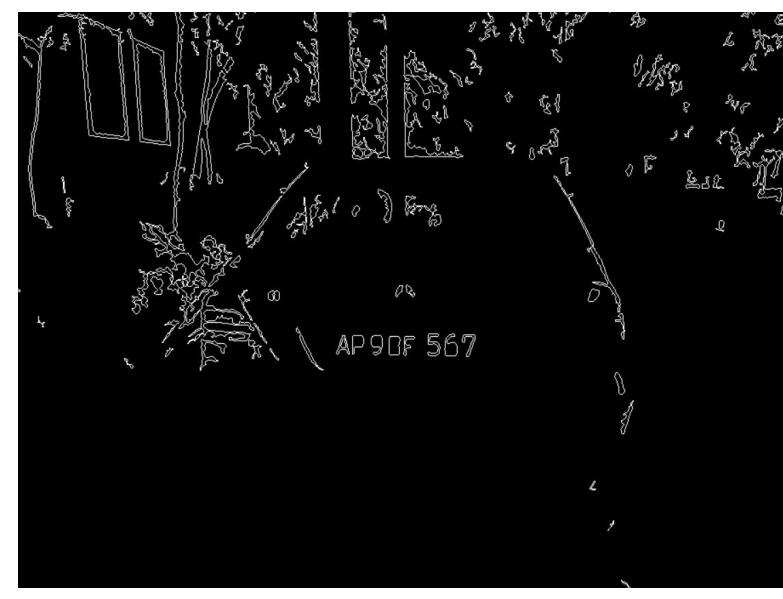

Fig-15

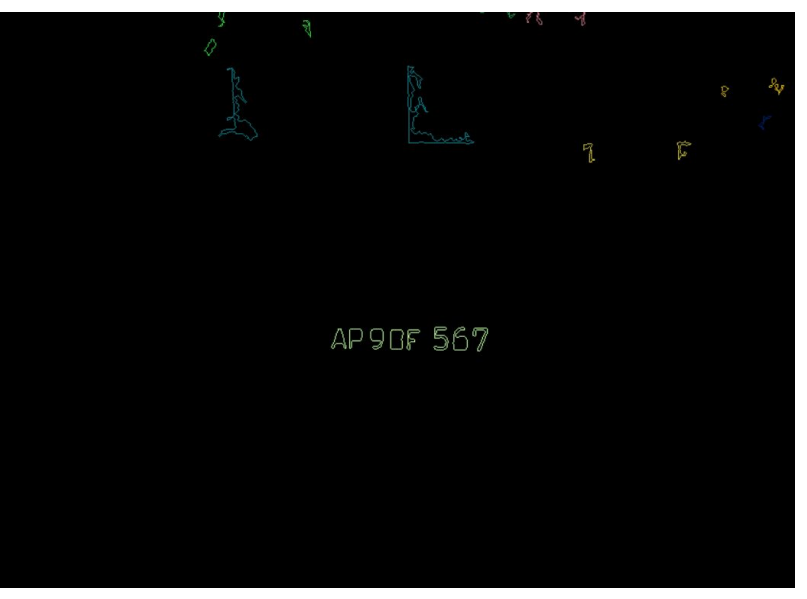

Fig-16

From the threshold images, features like lines, edges, ridges etc are separated to identify possible characters(shown in Fig-15). After the separation of all the lines, edges and ridges only detected character set is left out(Fig-16).

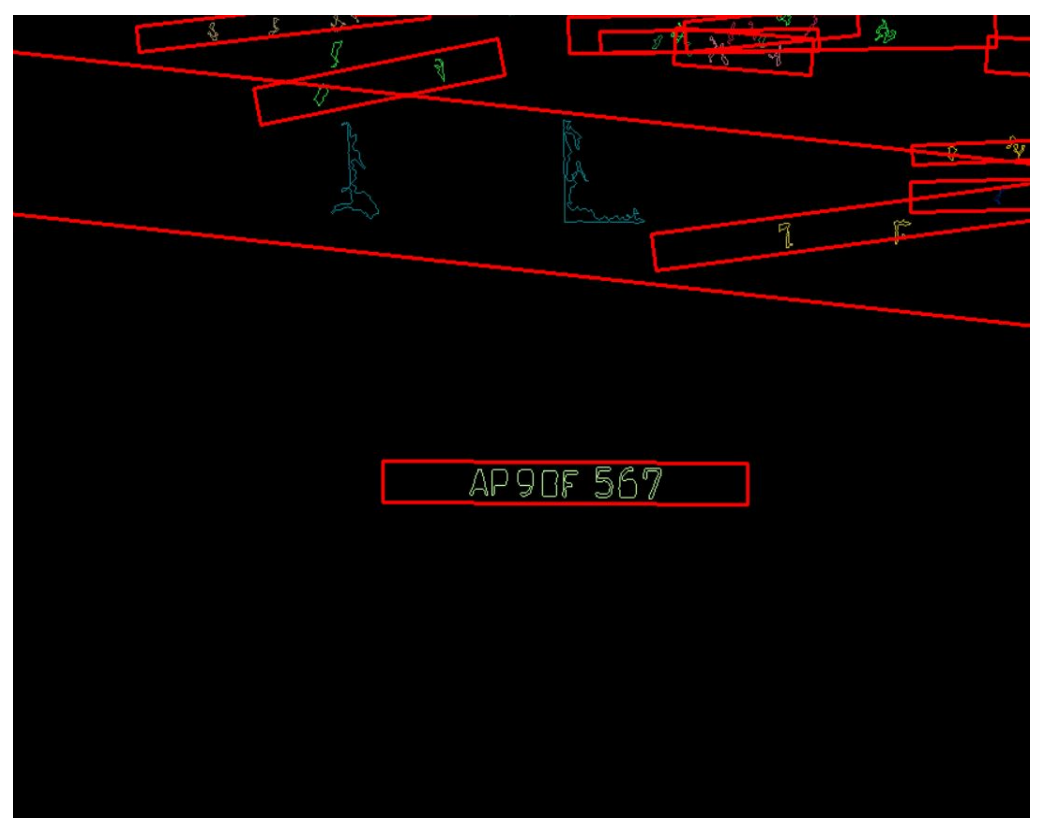

Fig-17

All the possible plates are identified and treated as rectangles, the rectangles which does not contain accurate characters will be discarded (Fig-17). 


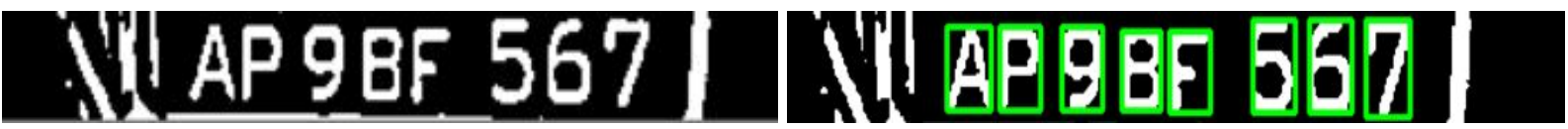

Fig-18

The final rectangle with appropriate characters from previous stage is matched with the trained characters individually (shown in Fig-18) and the extracted text is printed on the original image and also on the screen(as shown in Fig-19)
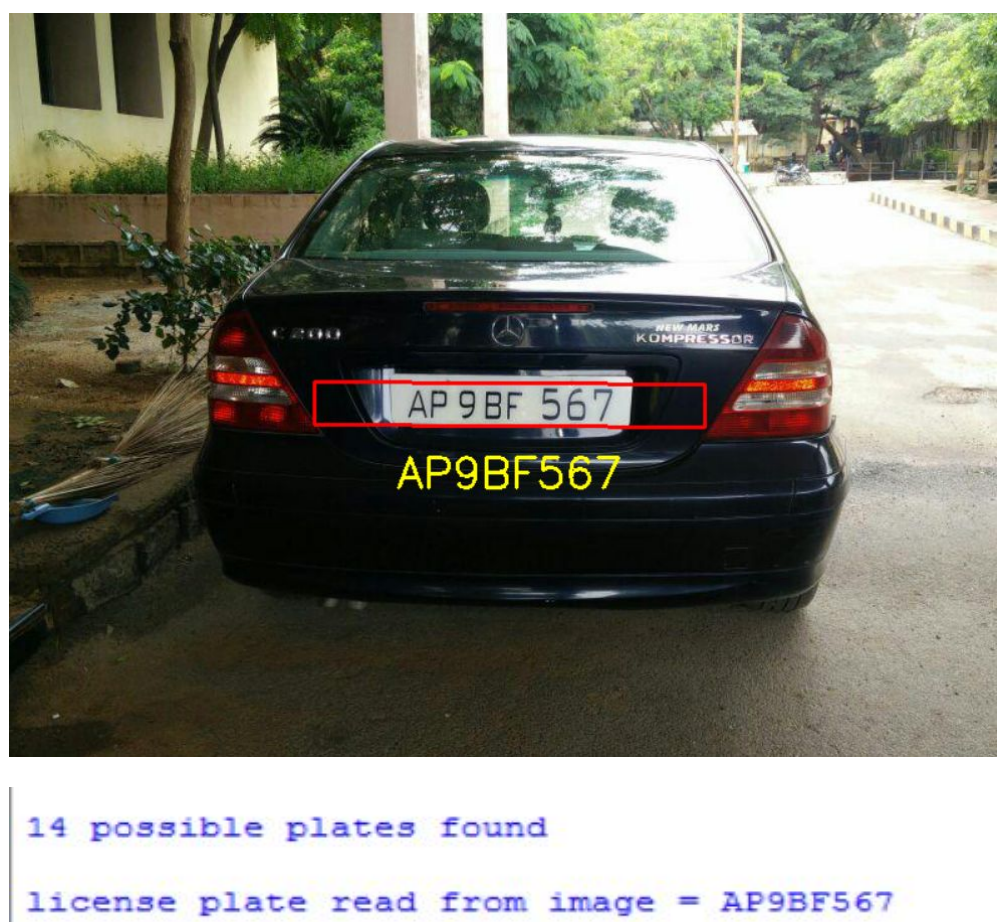

Fig-19 


\section{Test 2:}

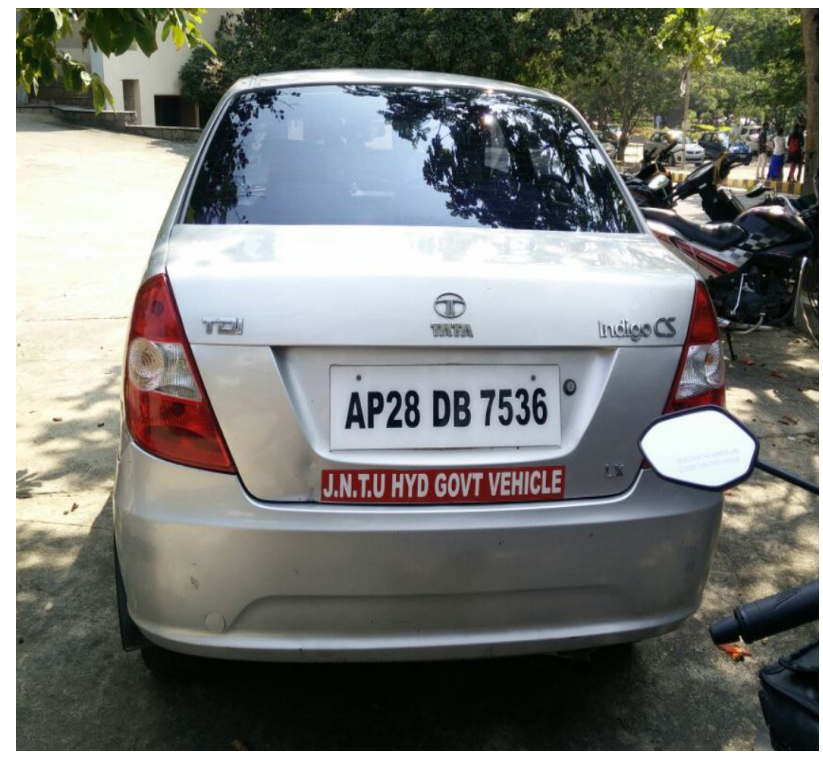

Fig-20

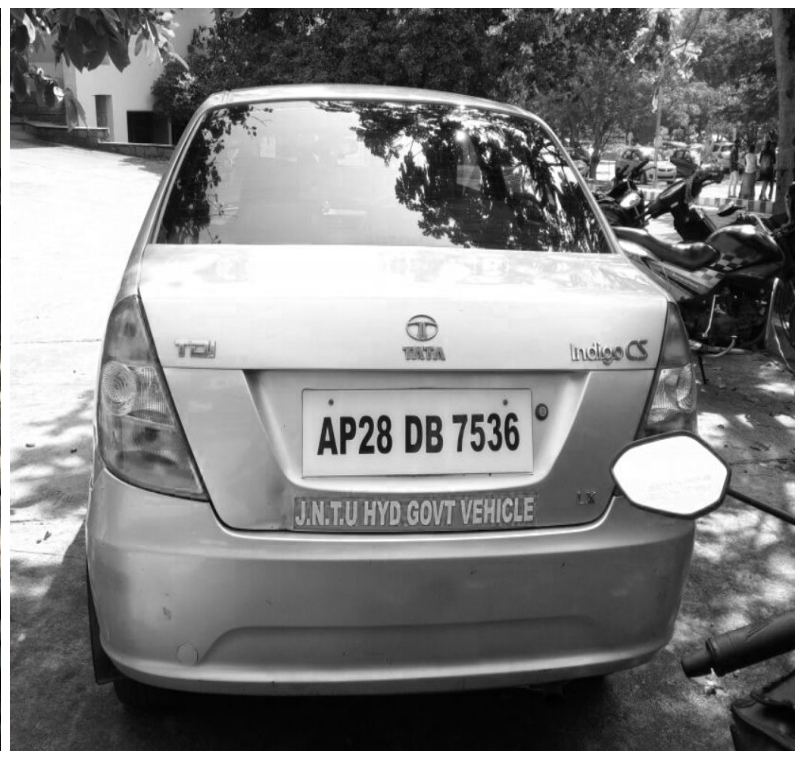

Fig-21

Fig-20 is original scene captured from webcam, From this image it is converted to Grayscale (Fig-21).

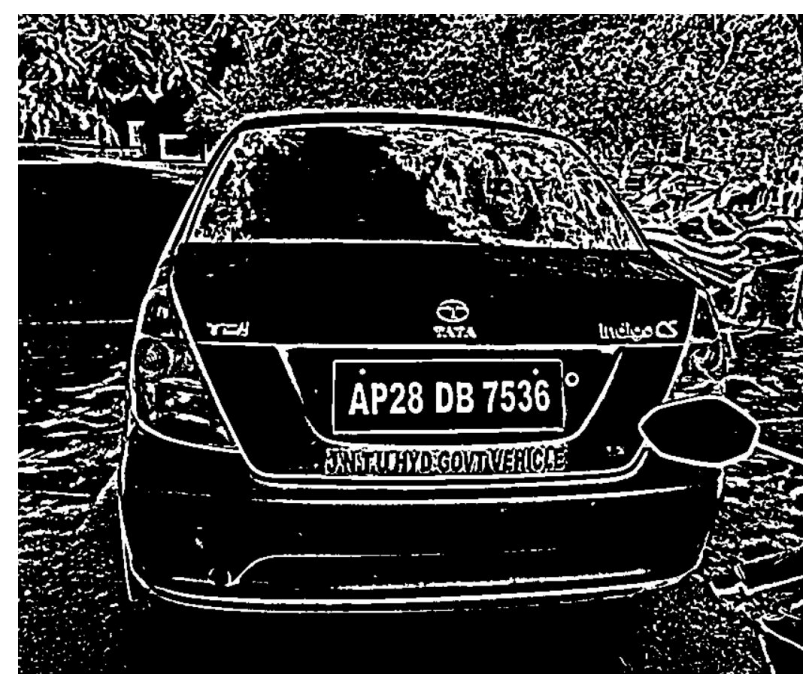

Fig-22

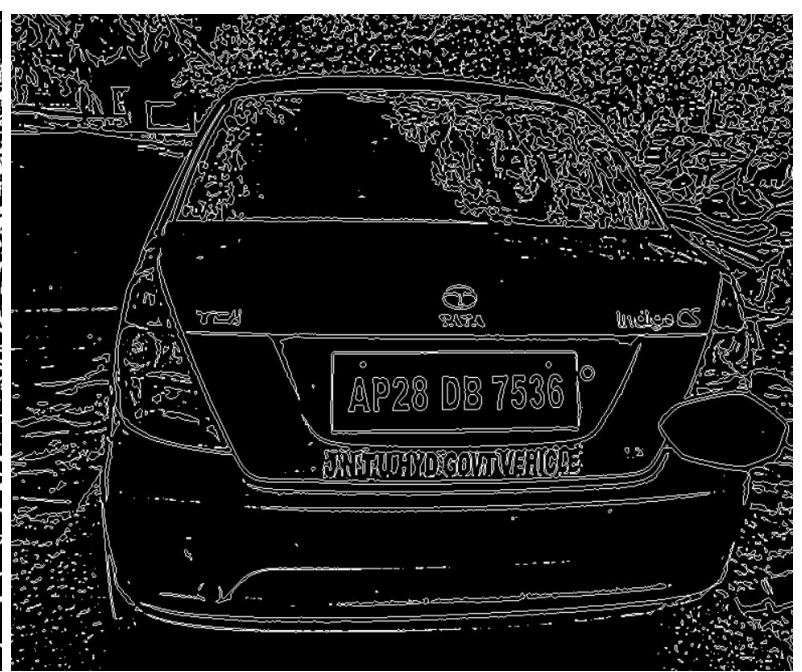

Fig-23

Threshold image(Fig-22) is obtained from grayscale image and it is further processed to to reduce noise(Fig-23). 


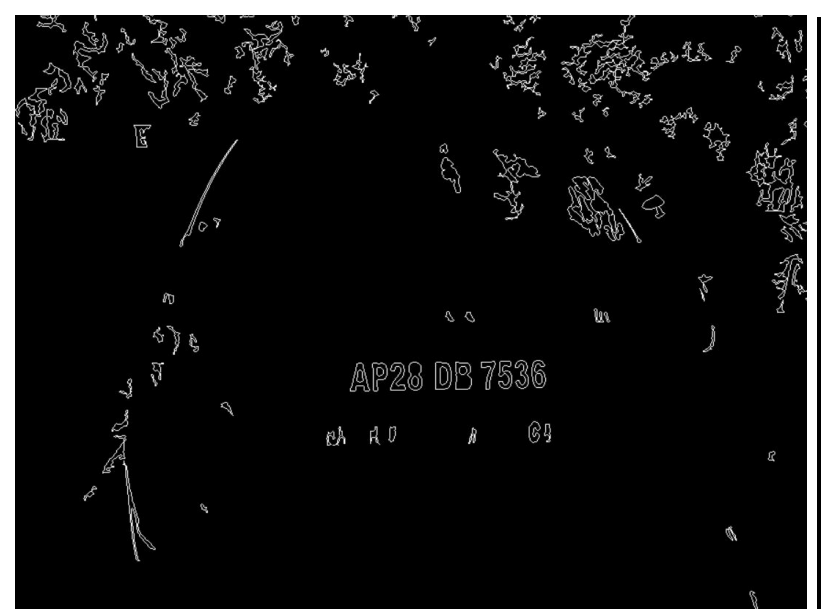

Fig-24

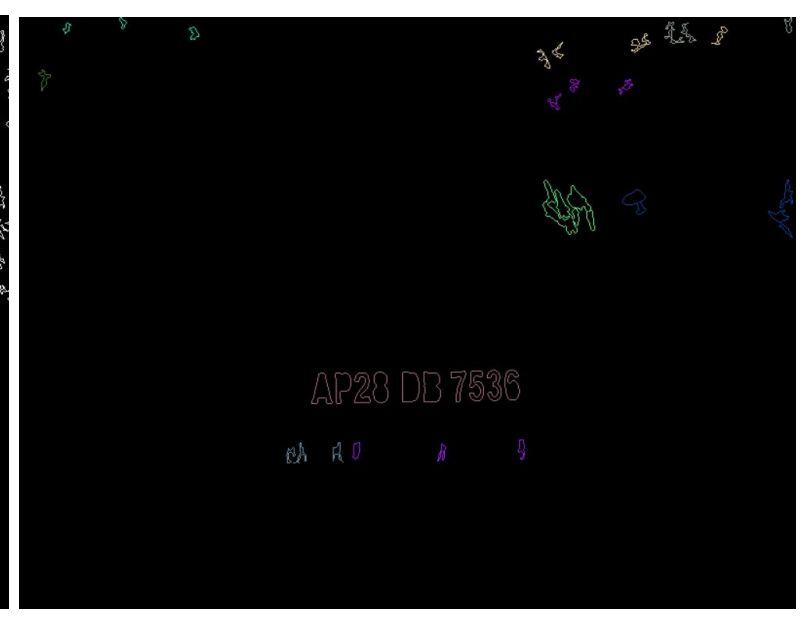

Fig-25

From the threshold images, features like lines, edges, ridges etc are separated to identify possible characters(shown in Fig-24). After the separation of all the lines, edges and ridges only detected character set is left out(Fig-25).

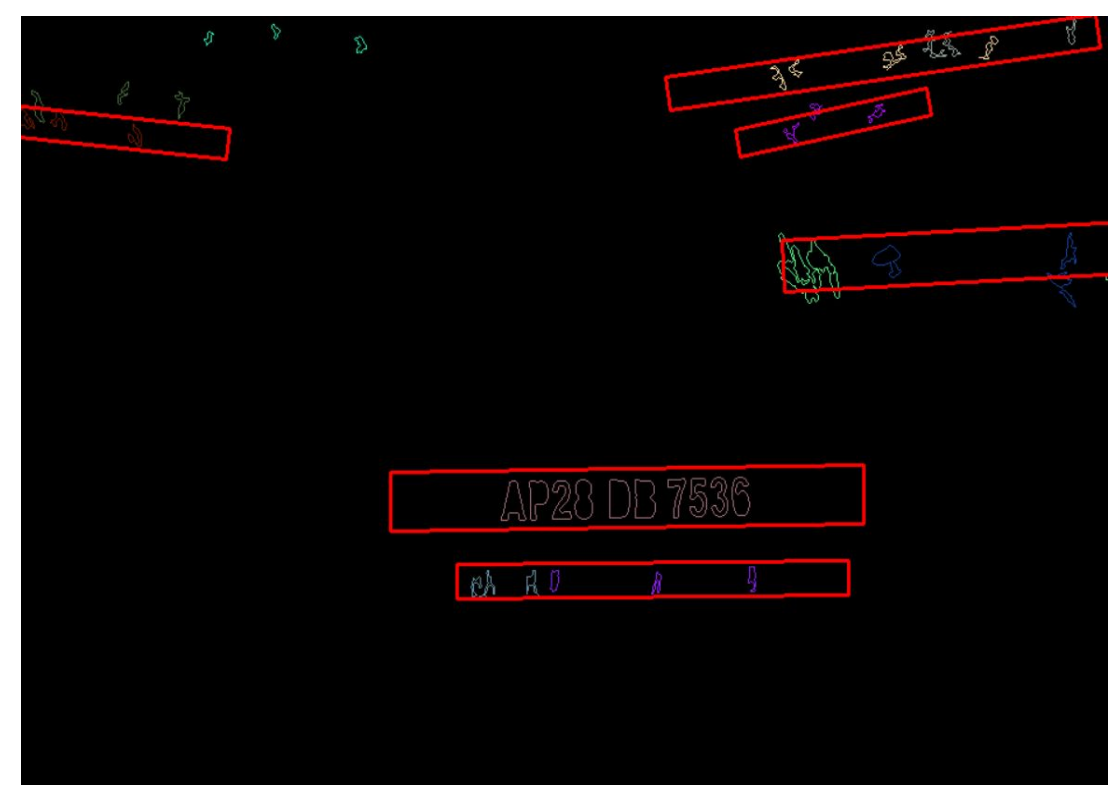

Fig-26

All the possible plates are identified and treated as rectangles, the rectangles which does not contain accurate characters will be discarded (Fig-26). 


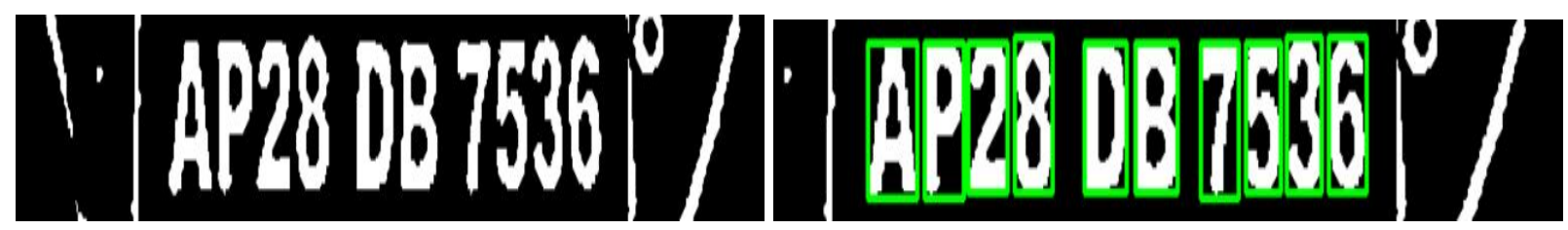

Fig-27

The final rectangle with appropriate characters from previous stage is matched with the trained characters individually (shown in Fig-27) and the extracted text is printed on the original image and also on the screen(as shown in Fig-28).
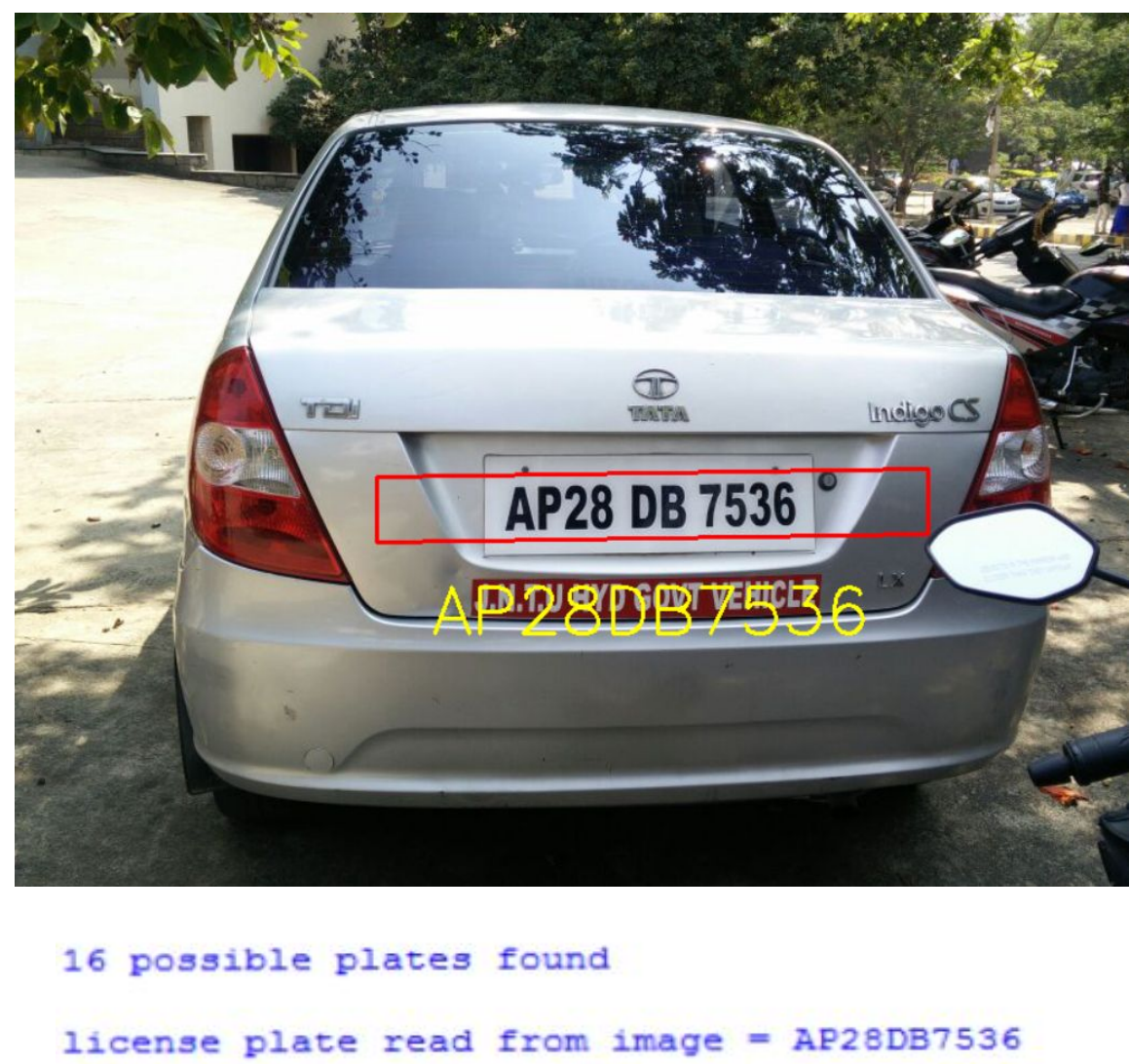

Fig-28 


\section{Test 3:}

The results shown below are of the source code taken directly from references before improvisation.

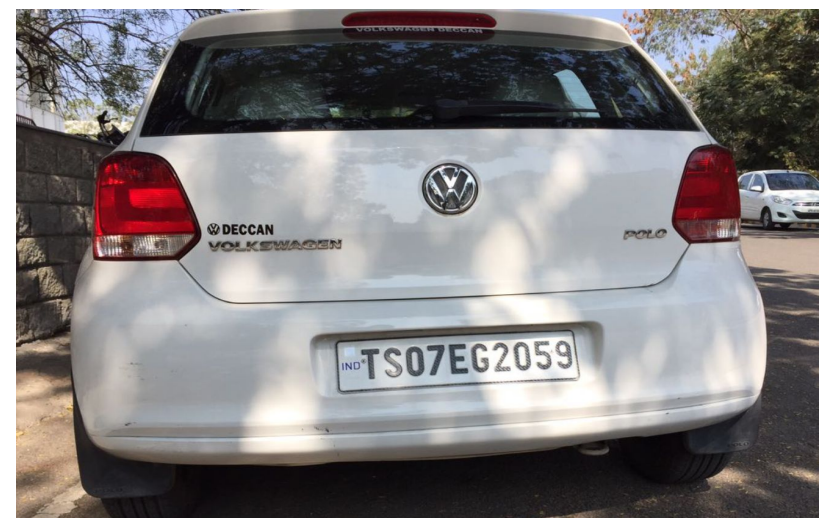

Fig-29

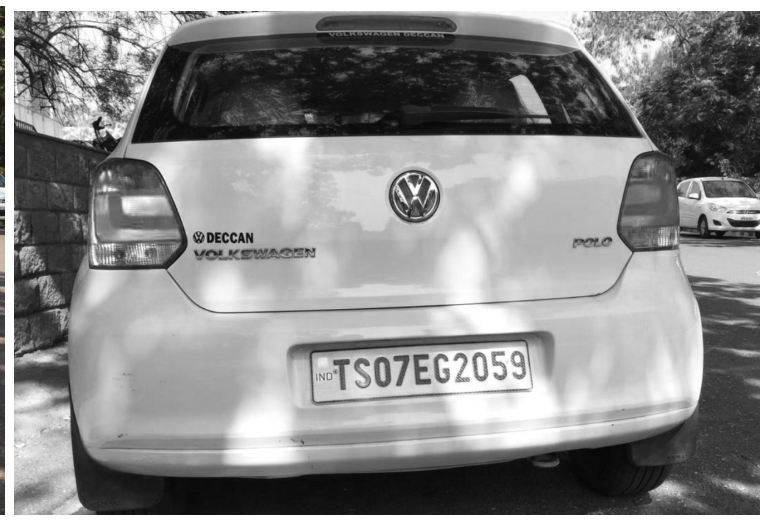

Fig-30

Fig-29 is original scene captured from webcam, From this image it is converted to Grayscale (Fig-30).

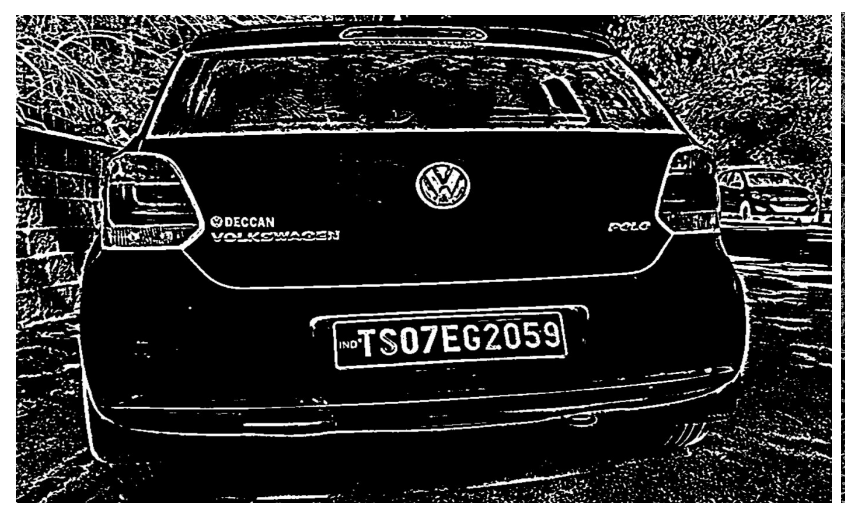

Fig-31

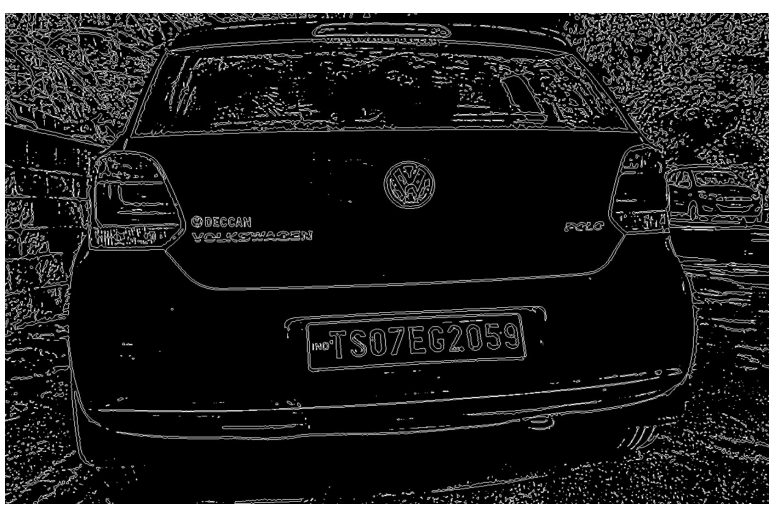

Fig-32

Threshold image(Fig-31) is obtained from grayscale image and it is further processed to to reduce noise(Fig-32). 


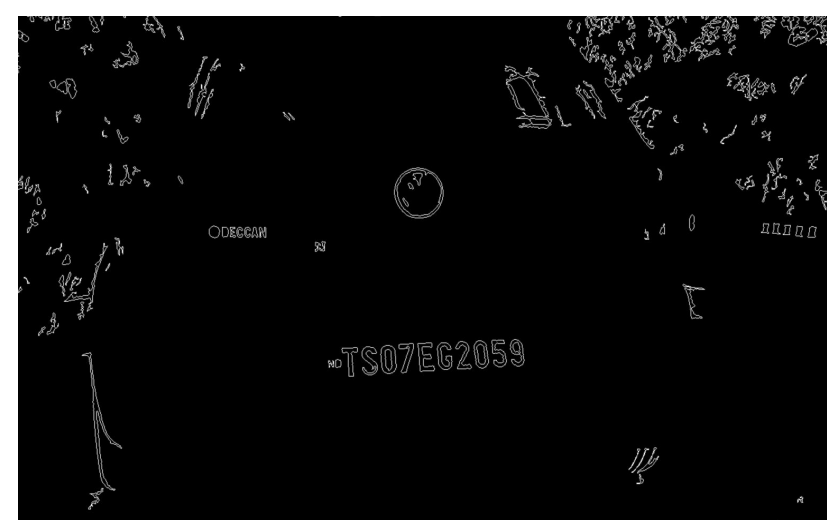

Fig-33

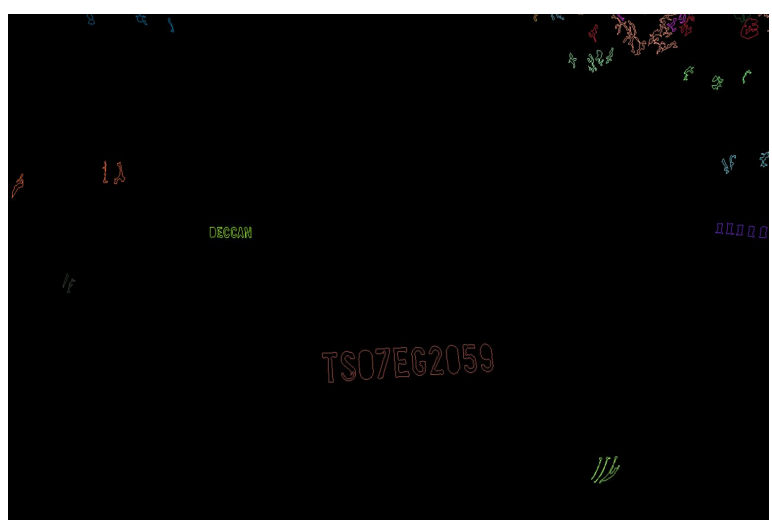

Fig-34

From the threshold images, features like lines, edges, ridges etc are separated to identify possible characters(shown in Fig-33). After the separation of all the lines, edges and ridges only detected character set is left out(Fig-34).

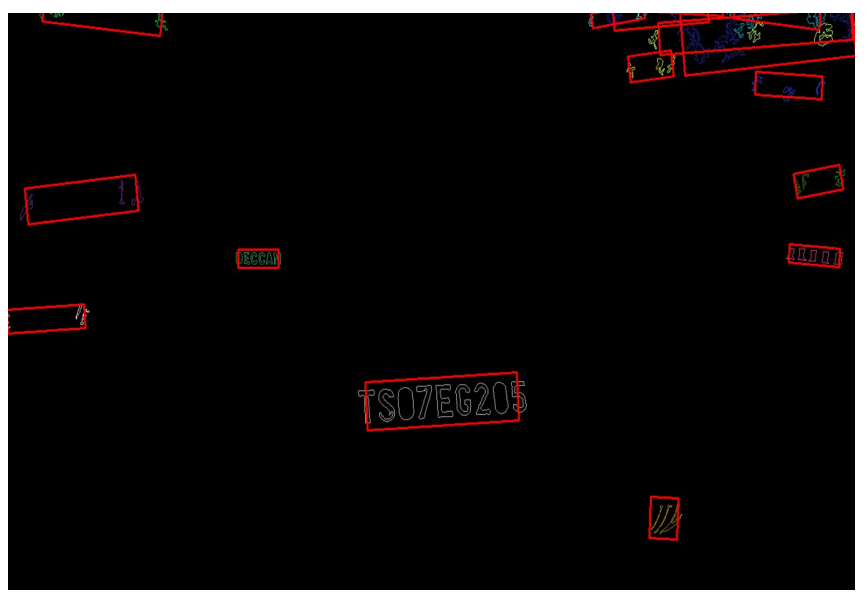

Fig-35

All the possible plates are identified and treated as rectangles, the rectangles which does not contain accurate characters will be discarded (Fig-35). 


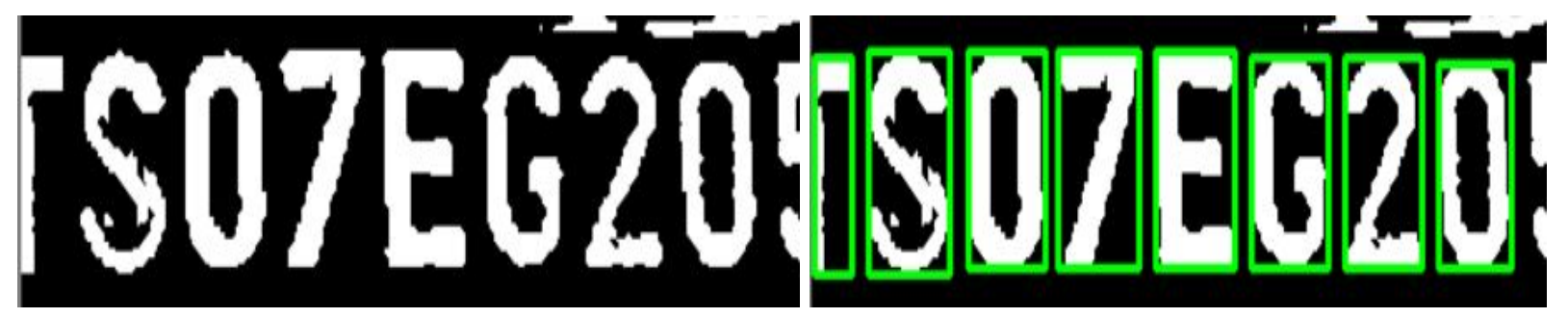

Fig-36

The final rectangle with appropriate characters from previous stage is matched with the trained characters individually (shown in Fig-36) and the extracted text is printed on the original image and also on the screen(as shown in Fig-37).

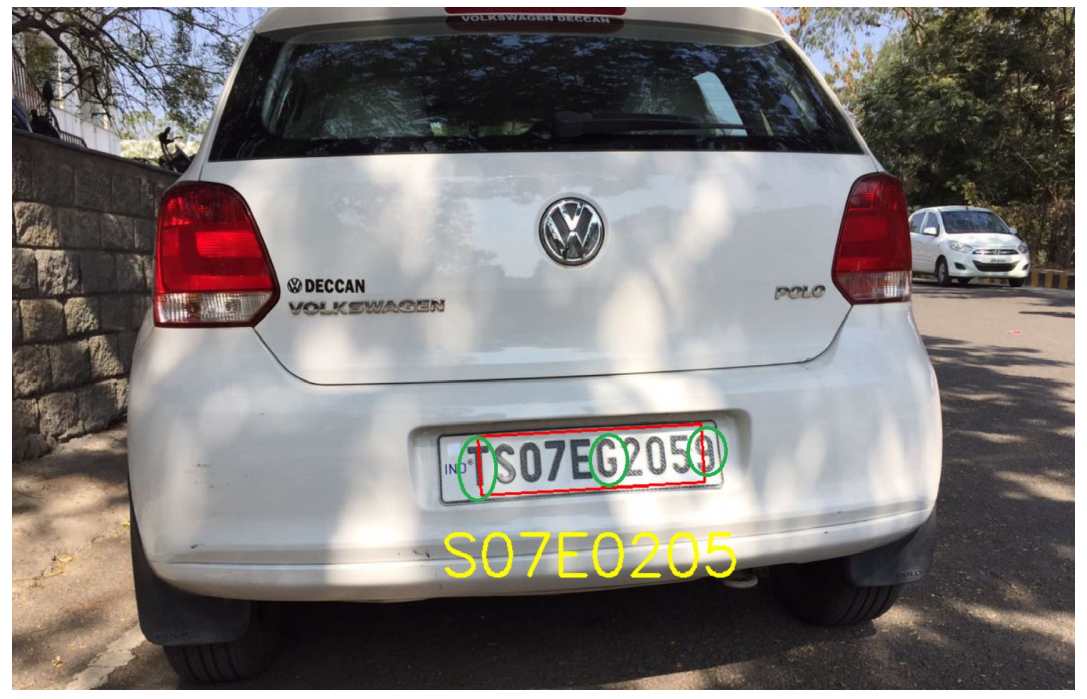

18 possible plates found

license plate read from image $=507 \mathrm{E} 0205$

Fig-37

In the above case only 8 characters are recognised from the given 10 characters input image, out of which only 6 are interpreted correctly. The border characters in the License Plate i.e "T \& 9" are not recognised because of dimensional difficulties for Indian standards. 
Recognition of text is still not $100 \%$ accurate even where clear imaging is available, Few characters which resemble similar to each other are printed incorrectly. Due to differences in font styles based on interest of vehicle owners there are slight misinterpretations in the extraction of characters. If the Government recommended standard license plate is used then the extraction of characters could be more accurate.

For example, $\mathrm{G}$ is misinterpreted as $\mathrm{O}, 6$ as 8,5 as $\mathrm{S}, 1$ as I,etc

Total accuracy can be achieved by human review.

\section{Test 4}

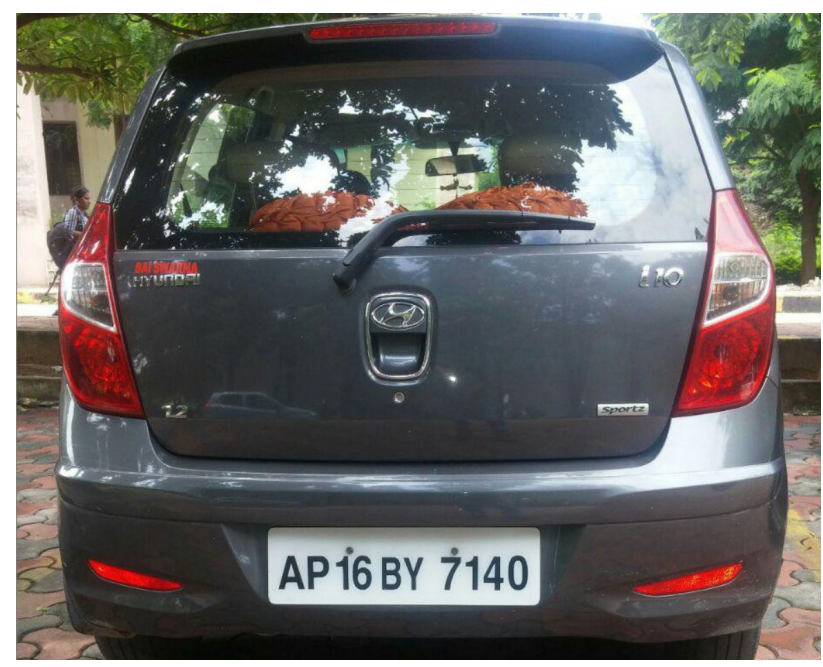

Fig-38

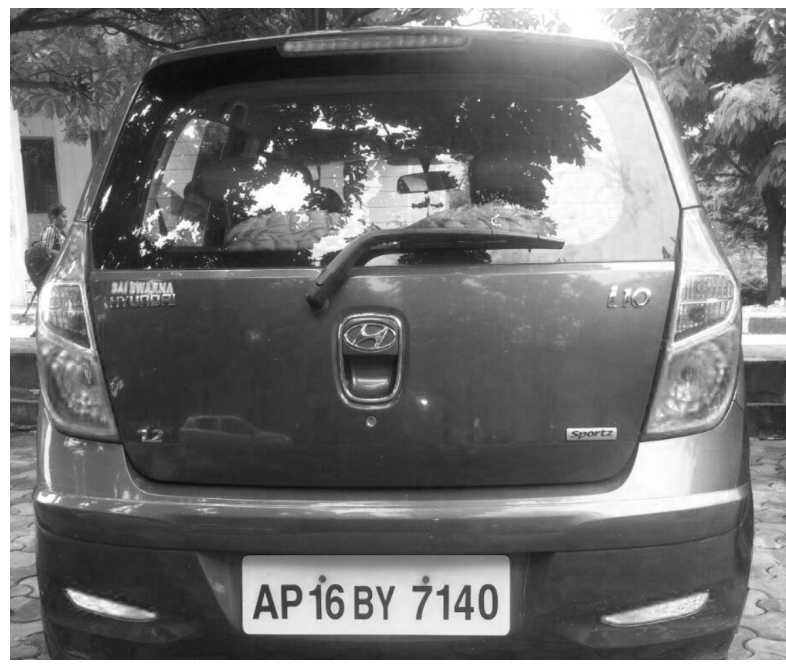

Fig-39

Fig-38 is original scene captured from webcam, From this image it is converted to Grayscale (Fig-39). 


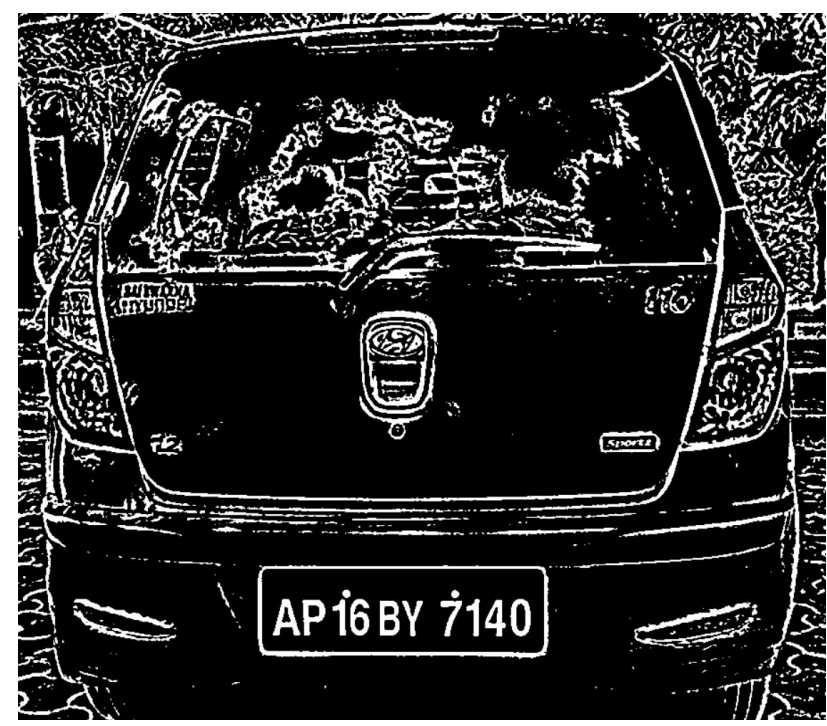

Fig-40

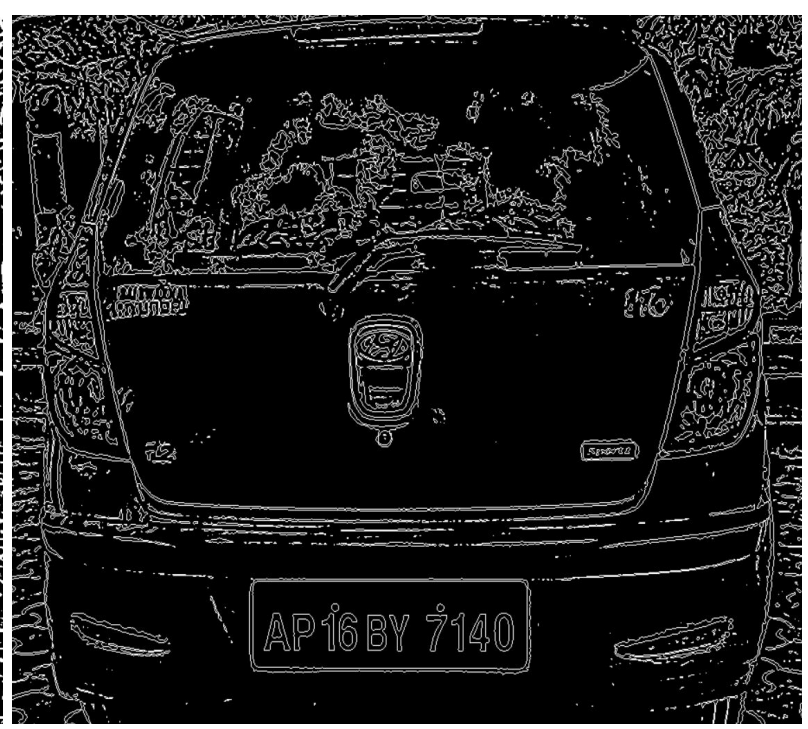

Fig-41

Threshold image(Fig-40) is obtained from grayscale image and it is further processed to to reduce noise(Fig-41).

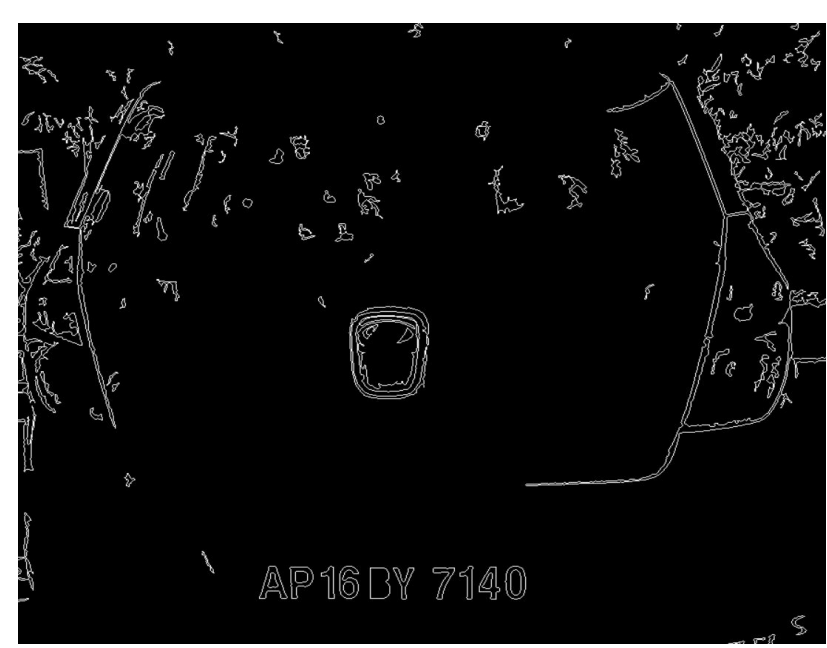

Fig-42

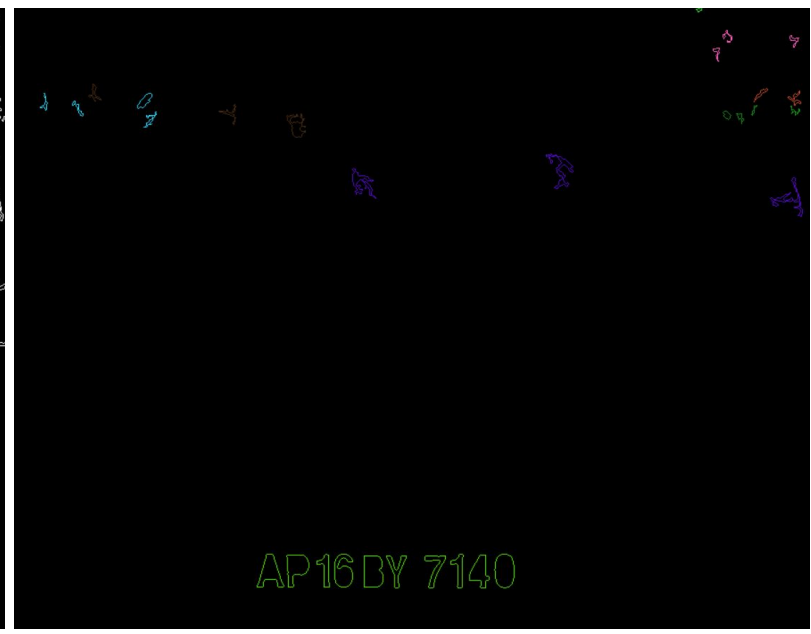

Fig-43

From the threshold images, features like lines, edges, ridges etc are separated to identify possible characters(shown in Fig-42). After the separation of all the lines, edges and ridges only detected character set is left out(Fig-43). 


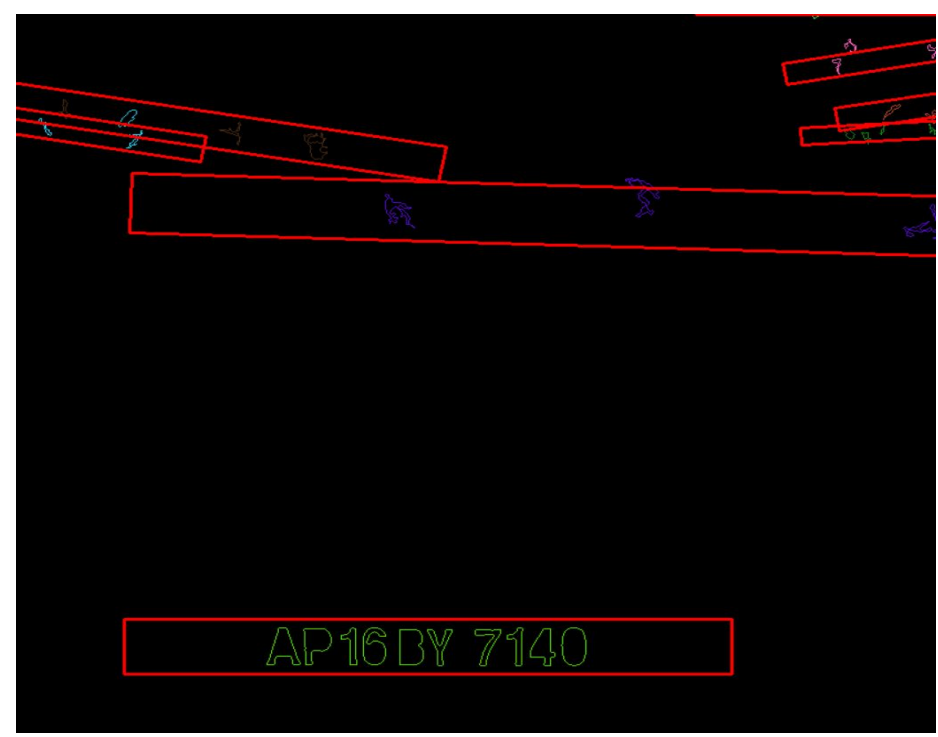

Fig-44

All the possible plates are identified and treated as rectangles, the rectangles which does not contain accurate characters will be discarded (Fig-44).

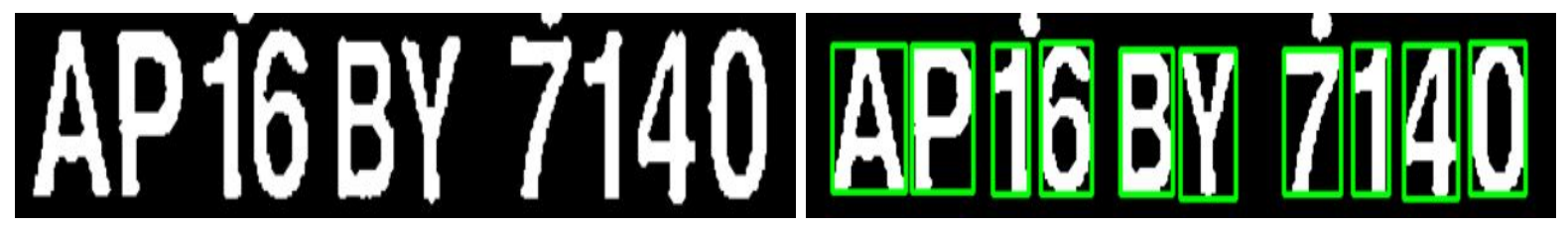

Fig-45

The final rectangle with appropriate characters from previous stage is matched with the trained characters individually (shown in Fig-45) and the extracted text is printed on the original image and also on the screen(as shown in Fig-46). 

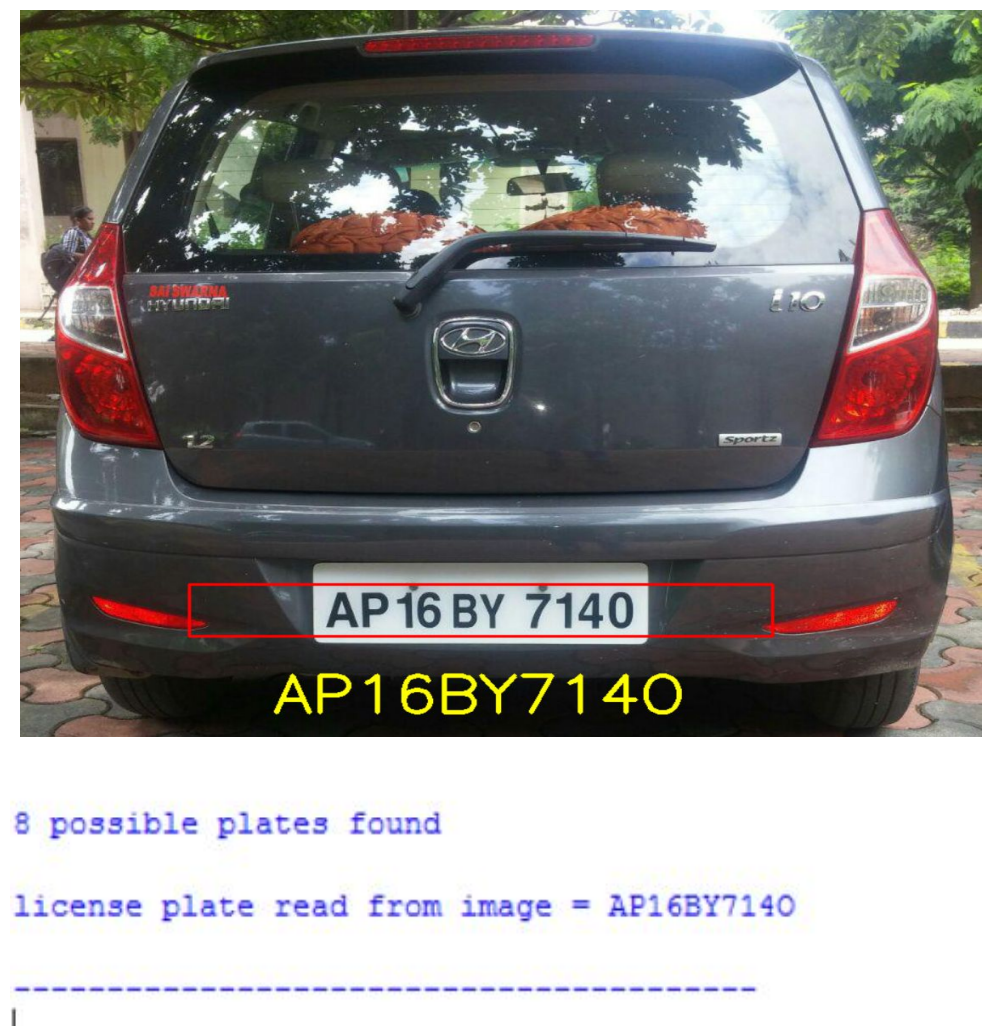

Fig-46

In this case the number " 0 " is misinterpreted as letter" $\mathrm{O}$ ". 


\section{Test 5:}

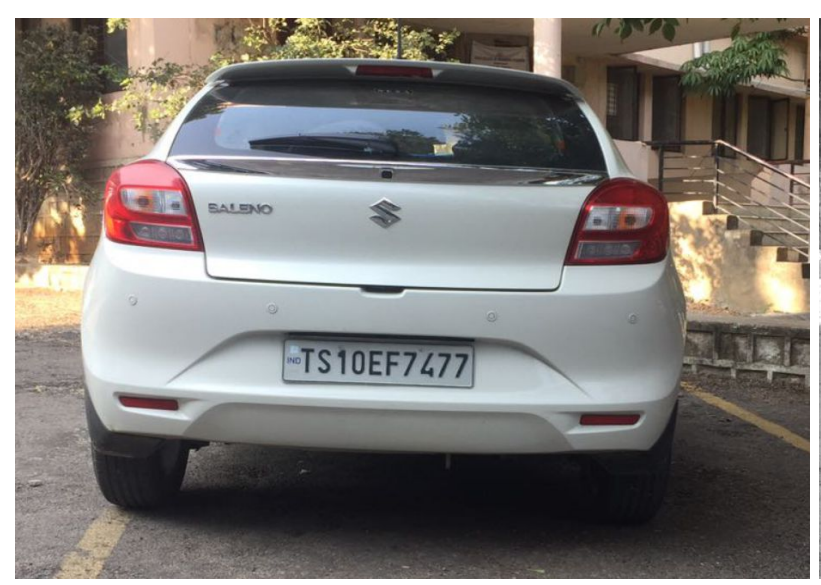

Fig-47

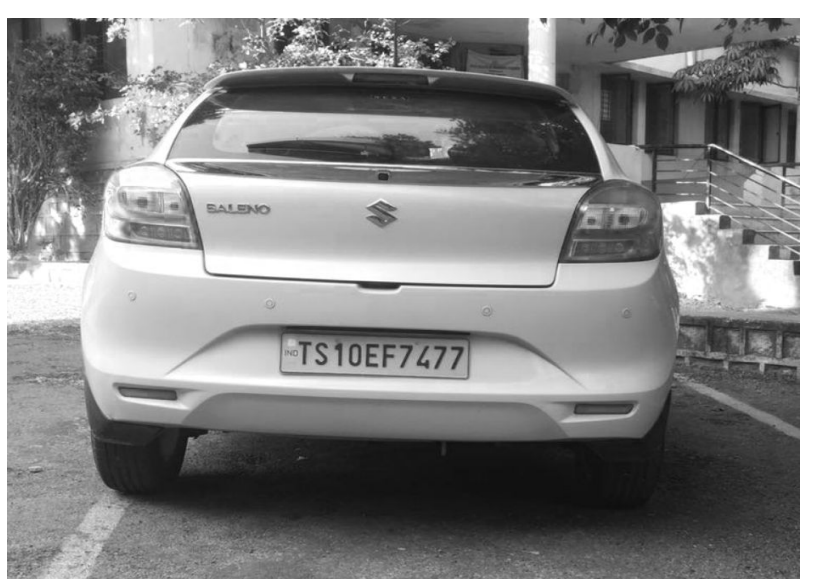

Fig-48

Fig-47 is original scene captured from webcam, From this image it is converted to Grayscale (Fig-48).

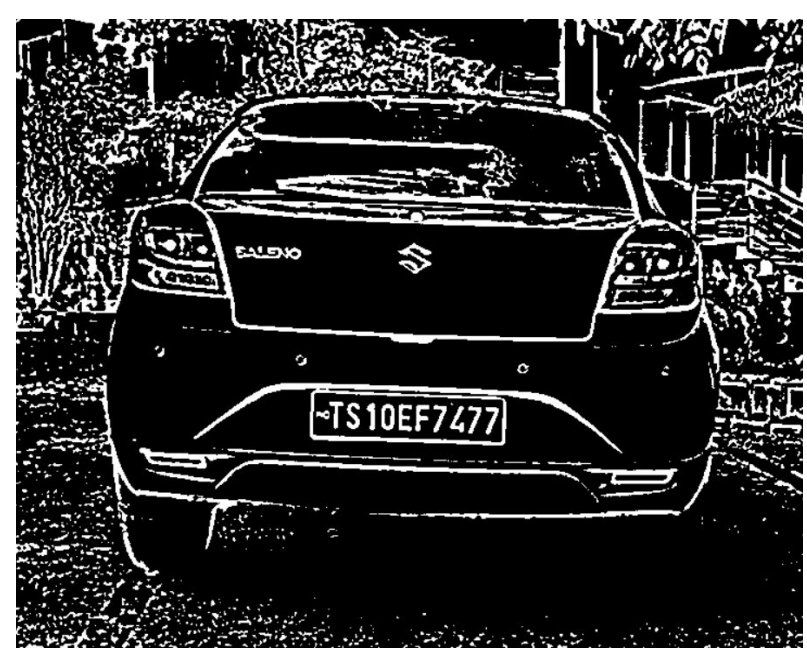

Fig-49

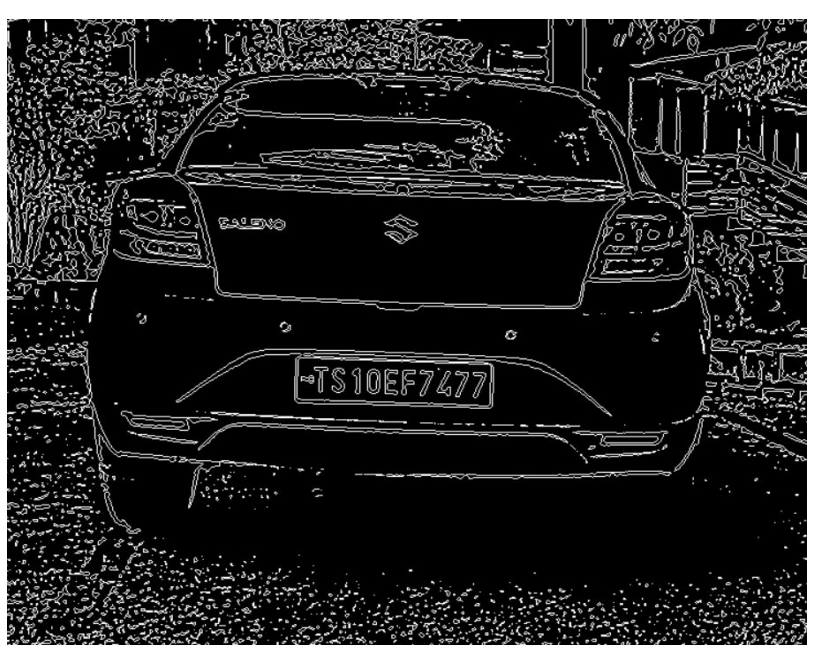

Fig-50

Threshold image(Fig-49) is obtained from grayscale image and it is further processed to to reduce noise(Fig-50). 


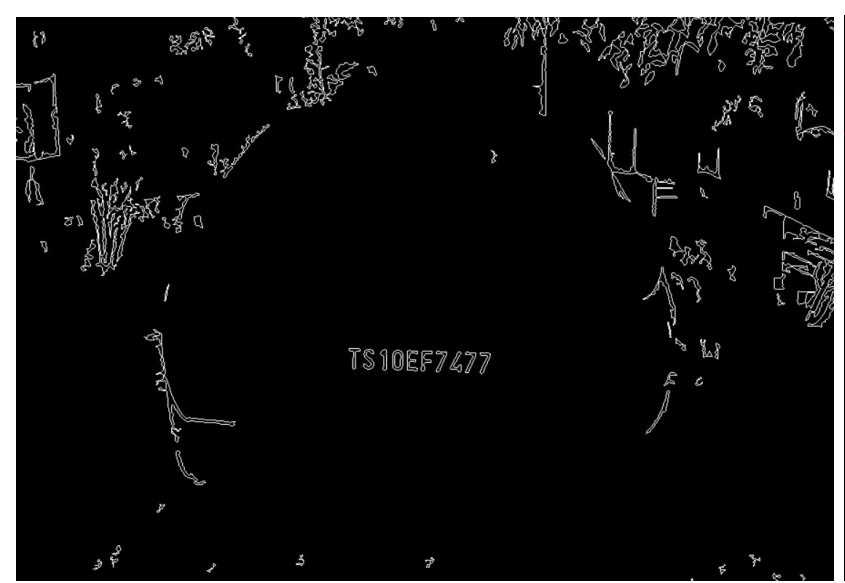

Fig-51

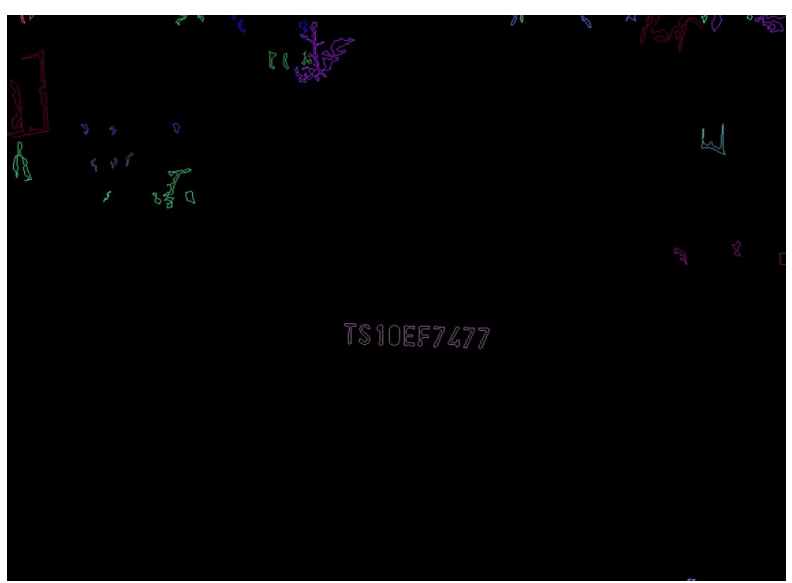

Fig-52

From the threshold images, features like lines, edges, ridges etc are separated to identify possible characters(shown in Fig-51). After the separation of all the lines, edges and ridges only detected character set is left out(Fig-52).

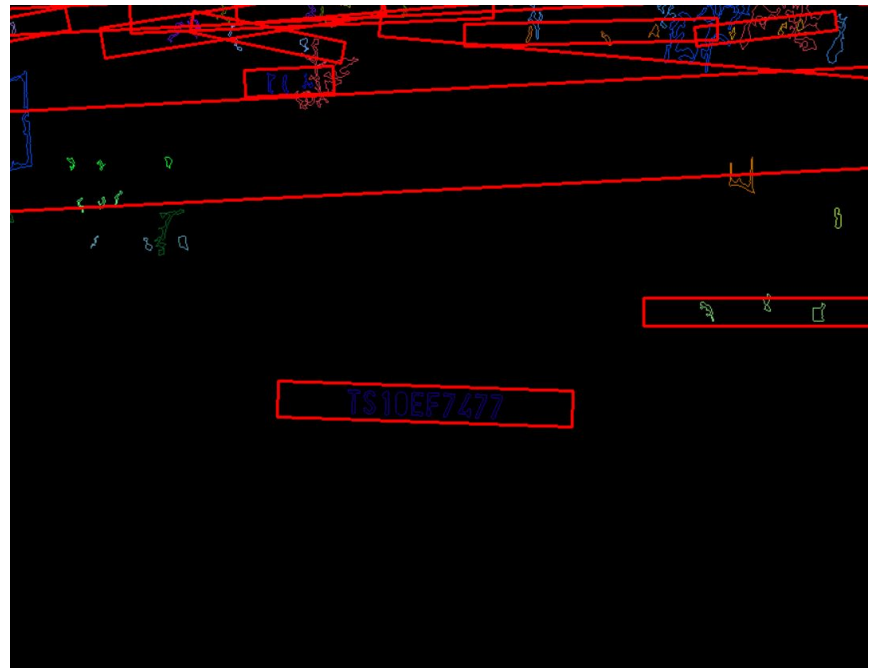

Fig-53

All the possible plates are identified and treated as rectangles, the rectangles which does not contain accurate characters will be discarded (Fig-53).
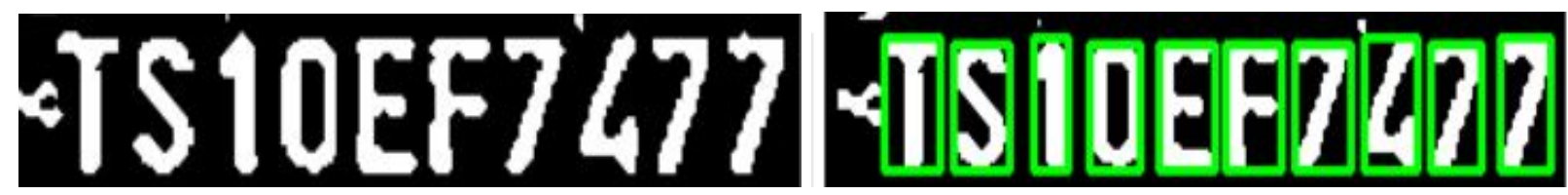

Fig-54 
The final rectangle with appropriate characters from previous stage is matched with the trained characters individually (shown in Fig-54) and the extracted text is printed on the original image and also on the screen(as shown in Fig-55).
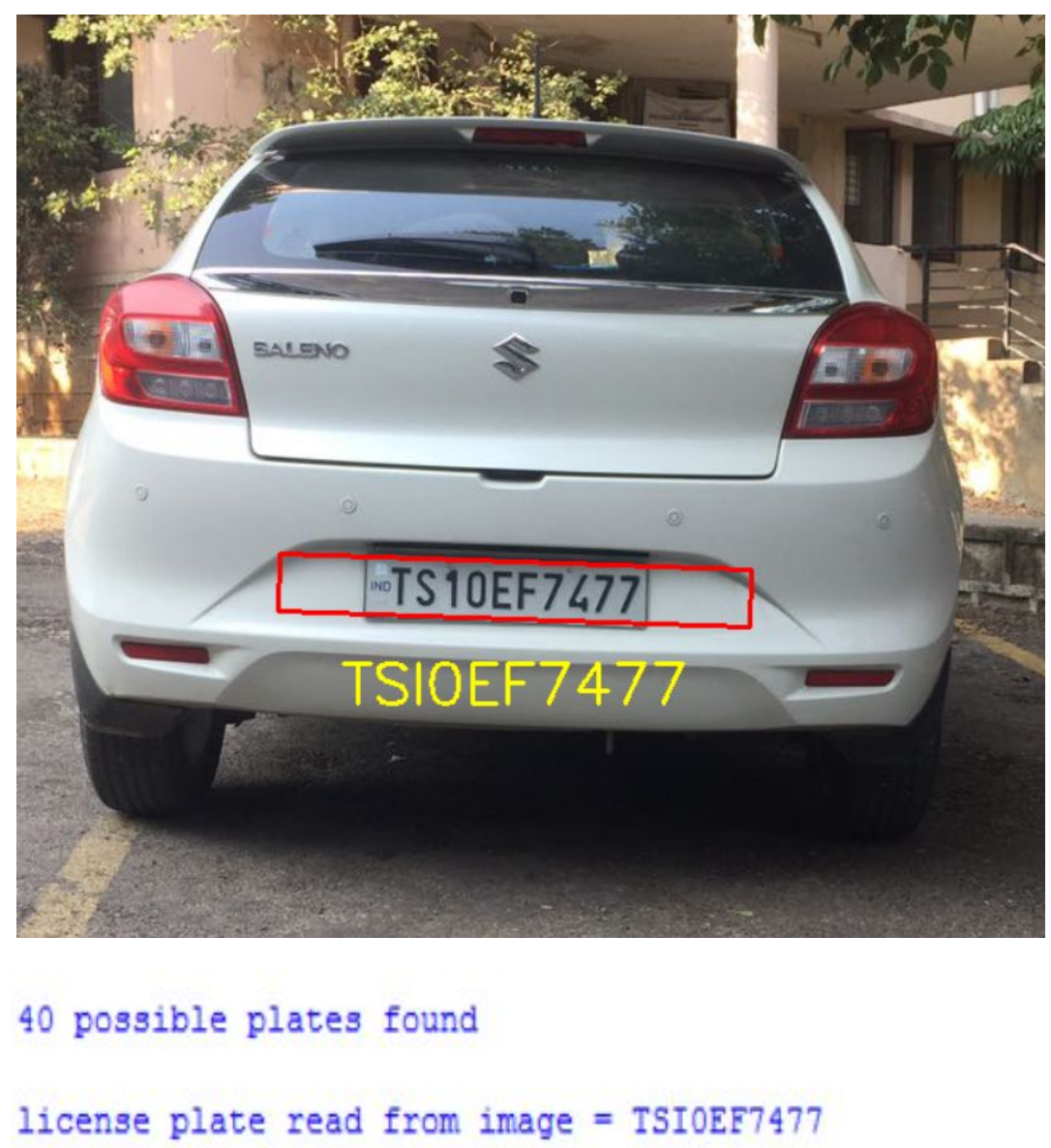

40 possible plates found

license plate read from image $=$ TSIOEF7477

Fig-55

In this case the number " 1 " is misinterpreted as letter " $I$ ".

\section{FUTURE SCOPE}

- Accuracy of recognition of License Plate can be increased further.

- Recognition of more than one License plate from a single image.

- RealTime implementation of system. 


\section{ACKNOWLEDGEMENTS}

We used the random images of license plates from the college. We are thankful to the people who cooperated to collect the data. We also used the Beaglebone Black open hardware from the Embedded Systems lab in the department. 


\section{REFERENCES}

1. Kulkarni, P., Khatri, A., Banga, P., \& Shah, K. (2009, April). Automatic number plate recognition (ANPR) system for indian conditions. In 2009 19th International Conference Radioelektronika (pp. 111-114). IEEE.

2. S.V. Rice, F.R. Jenkins, T.A. Nartker, The Fourth Annual Test of OCR

Accuracy, Technical Report 95-03. Information Science Research Institute, University of Nevada, Las Vegas,(1995).

3. Alexander J. Faaborg, Using Neural Networks to Create an Adaptive Character Recognition System, March 2002.

4. K.M. Sajjad, Automatic License Plate Recognition using Python and OpenCV. 


\section{APPENDIX}

\section{USER MANUAL:}

\section{Steps for installing Opencv 3.0 and Python 2.7:}

1.Open a terminal and update the apt-get package manager followed by upgrading any pre-installed packages:

\$ sudo apt-get update

\$ sudo apt-get upgrade

2.Now install developer tools:

\$ sudo apt-get install build-essential cmake git pkg-config

3.OpenCV needs to be able to load various image file formats from disk, including JPEG, PNG, TIFF, etc. In order to load these image formats from disk I/O packages are needed:

\$ sudo apt-get install libjpeg8-dev libtiff4-dev libjasper-dev libpng12-dev

4.GTK development library, which the highgui module of OpenCV depends on to build Graphical User Interfaces (GUIs) in order to display image on screen:

\$ sudo apt-get install libgtk2.0-dev

5.Images can be loaded using OpenCV, but for processing video streams and accessing individual frames this is needed:

\$ sudo apt-get install libavcodec-dev libavformat-dev libswscale-dev libv4l-dev

6. Install libraries that are used to optimize various routines inside of OpenCV:

\$ sudo apt-get install libatlas-base-dev gfortran

7.Install pip, a Python package manager:

\$ wget https://bootstrap.pypa.io/get-pip.py 
\$ sudo python get-pip.py

8.Install virtualenv and virtualenvwrapper, These two packages allow us to create separate Python environments for each project:

\$ sudo pip install virtualenv virtualenvwrapper

$\$$ sudo rm -rf $\sim /$ cache/pip

9. Now virtualenv and virtualenvwrapper are installed, update /.bashrc file:

export WORKON_HOME $=\$$ HOME $/$.virtualenvs

source/usr/local/bin/virtualenvwrapper.sh

\$ source $\sim /$.bashrc

10.create cv virtual environment where computer vision development and OpenCV $3.0+$ Python 2.7+ installation is done:

\$ mkvirtualenv cv

11.Now install Python 2.7 development tools:

\$ sudo apt-get install python2.7-dev

12. Since OpenCV represents images as multi-dimensional NumPy arrays,install NumPy into cv virtual environment:

$\$$ pip install numpy

13. Required environment is now all setup, change to home directory, pull down OpenCV from GitHub, and checkout the 3.0.0 version:

$\$ \mathrm{~cd} \sim$

\$ git clone https://github.com/Itseez/opencv.git

$\$$ cd opencv

$\$$ git checkout 3.0 .0 
14. opencv_contrib repo is needed as well. Without this repository, standard keypoint detectors and local invariant descriptors (such as SIFT, SURF, etc.) that were available in the OpenCV 2.4.X version cannot be accessed. Some of the newer OpenCV 3.0 features like text detection in natural images are also included in this:

$\$ \mathrm{~cd} \sim$

\$ git clone https://github.com/Itseez/opencv_contrib.git

\$ cd opencv_contrib

$\$$ git checkout 3.0 .0

15.Time to setup the build:

$\$$ cd $\sim /$ opencv

\$ mkdir build

$\$$ cd build

\$ cmake -D CMAKE_BUILD_TYPE=RELEASE ।

-D CMAKE_INSTALL_PREFIX=/usr/local ।

-D INSTALL_C_EXAMPLES=ON \

-D INSTALL_PYTHON_EXAMPLES=ON \

-D OPENCV_EXTRA_MODULES_PATH= /opencv_contrib/modules \

-D BUILD_EXAMPLES $=$ ON . .

16.Finally compile the OpenCV:

\$ make $-j 4$

17.Assuming that OpenCV compiled without error, Now install on the system:

\$ sudo make install

\$ sudo ldconfig

18. On reaching to this step without an error, OpenCV should now be installed in /usr/local/lib/python2.7/site-packages:

\$cd /.virtualenvs/cv/lib/python2.7/site-packages/

\$ In -s/usr/local/lib/python2.7/site-packages/cv2.so cv2.so 